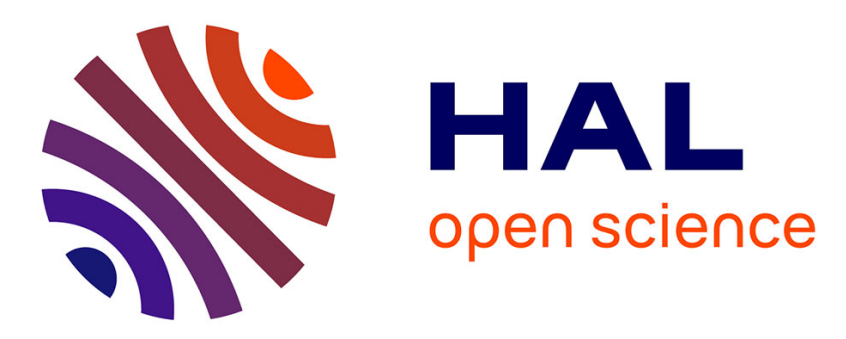

\title{
Collaborative Immersive Analytics
}

Mark Billinghurst, Maxime Cordeil, Anastasia Bezerianos, Todd Margolis

\section{To cite this version:}

Mark Billinghurst, Maxime Cordeil, Anastasia Bezerianos, Todd Margolis. Collaborative Immersive Analytics. Immersive Analytics, Marriott, K.; Schreiber, F.; Dwyer, T.; Klein, K.; Riche, N.H; Itoh, T.; Stuerzlinger, W.; Thomas, B.H., 2018, 978-3-030-01387-5. 10.1007/978-3-030-01388-2_8 . hal01938529

\section{HAL Id: hal-01938529 \\ https://hal.inria.fr/hal-01938529}

Submitted on 28 Nov 2018

HAL is a multi-disciplinary open access archive for the deposit and dissemination of scientific research documents, whether they are published or not. The documents may come from teaching and research institutions in France or abroad, or from public or private research centers.
L'archive ouverte pluridisciplinaire HAL, est destinée au dépôt et à la diffusion de documents scientifiques de niveau recherche, publiés ou non, émanant des établissements d'enseignement et de recherche français ou étrangers, des laboratoires publics ou privés. 


\title{
8. Collaborative Immersive Analytics
}

\author{
Mark Billinghurst ${ }^{1}$, Maxime Cordeil ${ }^{2}$, Anastasia Bezerianos ${ }^{3}$, and Todd \\ Margolis ${ }^{4}$ \\ 1 School of ITMS, University of South Australia, Mawson Lakes, Australia \\ mark.billinghurst@unisa.edu.au \\ 2 Monash University, Australia \\ max.cordeil@monash.edu \\ 3 Univ. Paris-Sud, CNRS, Inria, Université Paris-Saclay, France \\ anastasia.bezerianos@u-psud.fr \\ ${ }^{4}$ Qlik, California USA \\ tmargo@gmail.com
}

\begin{abstract}
Many of the problems being addressed by Immersive Analytics require groups of people to solve. This chapter introduces the concept of Collaborative Immersive Analytics (CIA) and reviews how immersive technologies can be combined with Visual Analytics to facilitate co-located and remote collaboration. We provide a definition of Collaborative Immersive Analytics and then an overview of the different types of possible collaboration. The chapter also discusses the various roles in collaborative systems, and how to support shared interaction with the data being presented. Finally, we summarize the opportunities for future research in this domain. The aim of the chapter is to provide enough of an introduction to CIA and key directions for future research, so that practitioners will be able to begin working in the field.
\end{abstract}

Keywords: collaborative immersive analytics, immersive analytics, immersion, human-computer interaction, visual analytics, collaboration, collaborative visualization, shared interaction

\subsection{Introduction}

In a world of increasing computing power and sensing, there is more data being generated than ever before. Over the last ten years Big Data [95] has become an important field of research with data scientists exploring many different ways to transform terabytes of information into valuable insights. One of the popular methods for creating understanding is to generate visual representations of the data, as explored in the area of Visual Analytics [125], defined as "the science of analytical reasoning facilitated by interactive visual interfaces". In this case a wide variety of visualization techniques are used to explore complex datasets. For example, 2D bar charts can be used to understand detailed election results and 3D graphics to show sensor data on terrain models. Visualization tools have been shown to improve performance time and productivity on a range of different tasks 
such as software analysis [50], data analysis [118], and information retrieval [130], among others. Sun et al. [119] provide an excellent summary of visual analytics techniques and applications, and their benefits.

Most recently, researchers have begun to explore how novel immersive display technology can be used to enhance visual analytics solutions (Chapter ??). The term Immersive Analytics [20] was coined to describe "an emerging research thrust investigating how new interaction and display technologies can be used to support analytical reasoning and decision making". These display technologies range from room scale immersive CAVE projection systems [30] to Virtual Reality head mounted displays (HMDs) [31], and from interactive walls, tables and multidisplay environments [124] to portable head worn Augmented Reality displays [3]. Klapperstück et al. [72] proposed ContextuWall, a system for interactive local and remote collaboration using touch and mobile devices as well as displays of various sizes. Emerging interaction technologies include devices like the Microsoft Kinect [71] or Leap Motion [74] which support natural gesture input, eye-tracking devices [99] for collecting awareness information, and even Brain Computer Interfaces that respond to thoughts [87].

Previous research has shown that using Immersive Analytics technologies can enable people to be significantly more effective at understanding data visualizations than more traditional interface technologies. For example, Belcher et al. found that using Augmented Reality (AR) improved user performance at finding node connections in complex graph visualizations compared to a $2 \mathrm{D}$ desktop presentation [8]. Similarly, Ware [135] found that using head-coupled stereo viewing enabled a person to understand an abstract graph three times the size of a graph viewed on a normal non-stereo monitor.

However, effective presentation on immersive displays is only one way to gain an understanding of complex data. Research stretching back over decades has found that collaborative decision making is often more effective than working on problems alone. Hill [51] provides a good review of early research comparing group versus individual performance on different tasks, finding that group performance is generally superior to that of an average individual. Similarly, in comparing collaborative to single user performance on an information visualization task, Mark et al. [83] found that groups worked slower, but produced more accurate results. Recently, Woolley et al. [137] have argued for a group collective intelligence factor that predicts performance on collaborative tasks, and could be improved by using collaborative tools. However, supporting effective collaboration can be challenging and was identified as one of the Grand Challenges for Visual Analytics [27].

Co-located collaboration provides important benefits. Sawyer et al. showed that team rooms supporting face-to-face activities helped focus the activities of work groups and removed them from interruptions [108]. Most recently, Teasley et al. found that co-located software teams working in "war rooms" with access to tools such as computers, whiteboards and flipcharts were twice as productive as the similar teams working in a traditional office environment [123]. Some 
visualization systems, such as CoVis [33] have specific tools for supporting co-located collaboration.

Significant benefits can be found from teams working together in an Immersive Analytics setting. Marai et al. [82] review three Immersive Analytics projects undertaken by research teams using the CAVE2 immersive projection environment (see Figure 1). The feedback was overwhelmingly positive, with the authors saying, "At the end of the meeting one of the team members said that the team got more done in 2 days than in 6 months of email, Skype, and Google Hangout." They were able to make such rapid progress because the CAVE2 environment enabled a group of people to work together face to face while seeing multiple representations of the data across a large amount of screen real-estate all at the same time. This research builds on their earlier work with the Cyber-Commons [76] which was a 100 megapixel tiled display wall that allowed small groups of multidisciplinary researchers to work together.

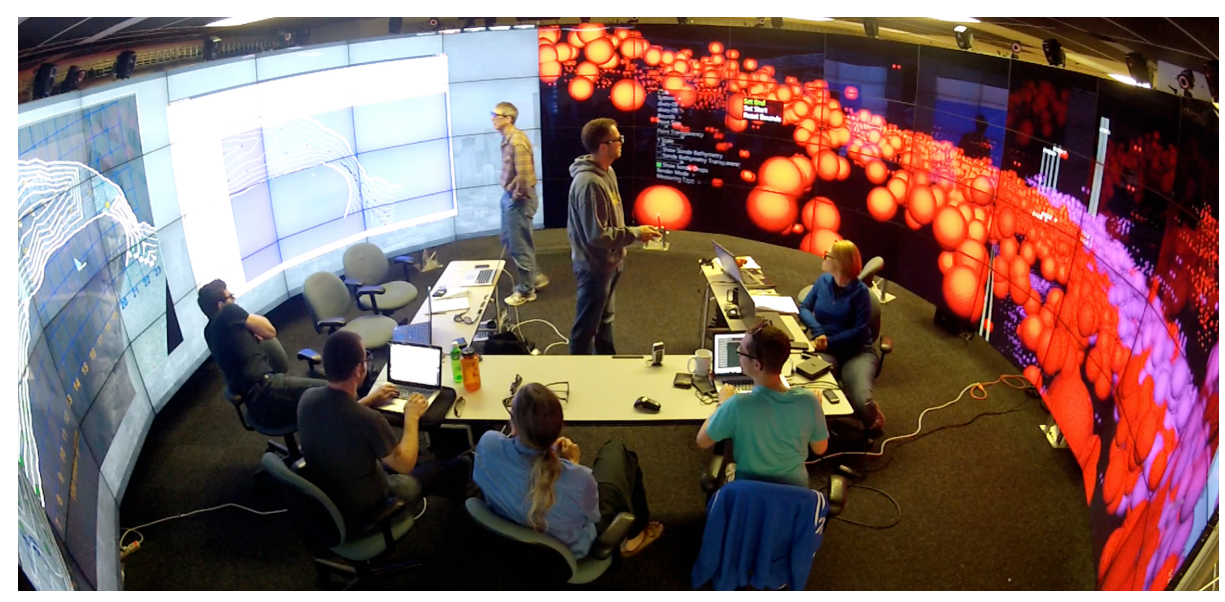

Fig. 1: EVL researchers at work in the CAVE2 at UIC, from [82]

In this chapter we provide an overview of and introduction to Collaborative Immersive Analytics. Although there has been a significant amount of research on how immersive technologies can support collaboration, there has been very little research in the newer area of Collaborative Immersive Analytics. Our aim is to provide an introduction to researchers to this field, describe some example applications, provide design guidelines, and clearly identify directions for future research. This should be helpful for guiding researchers wanting to enter this emerging field of research or for teams requiring new means of analysis for complex data.

In the remainder of the chapter we first provide a definition of Collaborative Immersive Analytics, and various roles people have when using collaborative systems. We then provide an overview of collaborative interaction methods, before 
identifying promising areas for research. Finally, we summarize the chapter and outside areas for future work. Overall, the aim of this chapter is to provide enough of an introduction to Collaborative Immersive Analytics that interested readers could begin to do their own work in the field.

\subsection{Definition and Scope}

Before we can discuss Collaborative Immersive Analytics (CIA) we need to define what the term means and describe the overall scope of the chapter. CIA is so new that there is no well accepted definition. However Collaborative Immersive Analytics is related to Collaborative Visualization, a term that Isenberg et al. [58] define as:

The shared use of computer-supported, (interactive,) visual representations of data by more than one person with the common goal of contribution to joint information processing activities.

Using this definition, Isenberg points out that collaborative visualization lies at the intersection of the two major research fields of visualization and computer supported collaborative work (CSCW), each of which have made significant research contributions that could benefit collaborative visualization.

Considering this and returning to the original definition of Immersive Analytics, we define Collaborative Immersive Analytics as: The shared use of immersive interaction and display technologies by more than one person for supporting collaborative analytical reasoning and decision making.

The main difference between Collaborative Immersive Analytics and Collaborative Visualization is the focus in CIA on the use of immersive interaction and display technologies. Hackathorn and Margolis [45] point out that Immersive Analytics environments can span most of Milgram's Reality-Virtuality (RV) continuum [86]. The RV continuum is a well-known way of classifying interface technologies in terms of how they connect the physical and virtual worlds, from the fully physical world (on the left) to the fully virtual world (on the right) (Figure 2). Within this continuum is the class of Mixed Reality technologies which merge real and virtual worlds. This ranges from tabletop displays and Augmented Reality environments to fully immersive virtual reality head mounted displays and rooms.

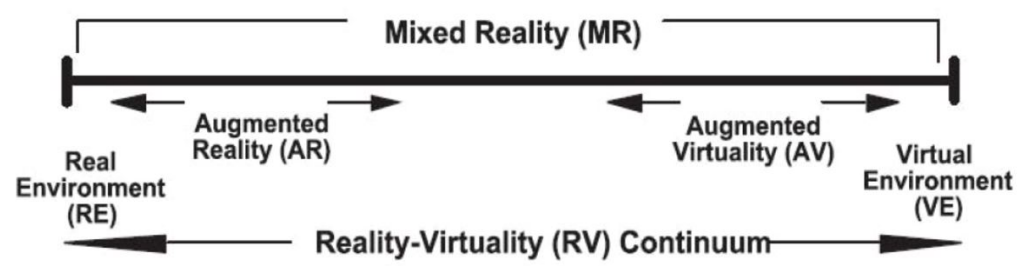

Fig. 2: Milgram's Mixed Reality Continuum, from [86]. 
Collaborative Immersive Analytics explores how Mixed Reality technologies such as these can be used in a collaborative setting. In contrast, Collaborative Visualization is concerned more with shared visualization in general, so the field of CIA can be viewed as a subset of the broader field of Collaborative Visualization. Just as Collaborative Visualization lies at the intersection of the field of visualization and CSCW, Collaborative Immersive Analytics lies at the intersection of Collaborative Visualization and Mixed Reality (see Figure 3).

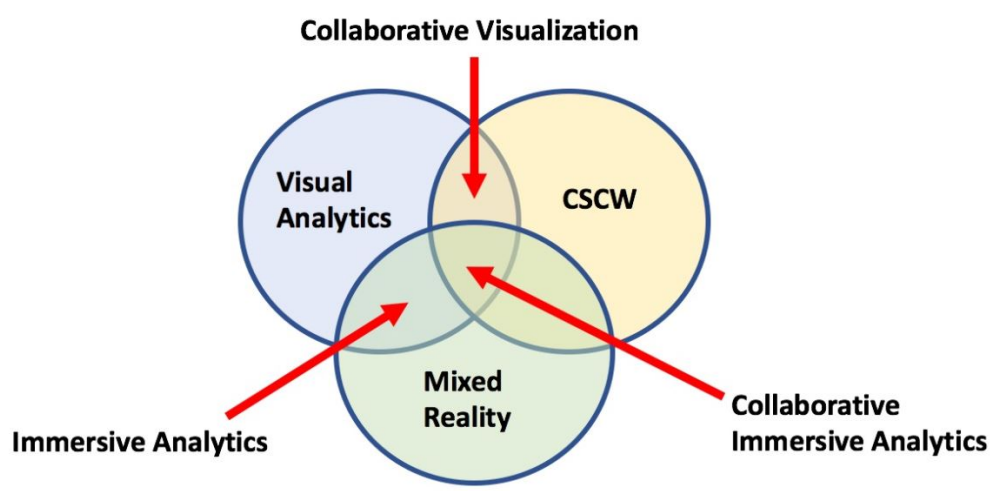

Fig. 3: The relationship between Collaborative Immersive Analytics, Collaborative Visualization and Immersive Analytics.

By its very nature, CIA is a multi-disciplinary research field. In addition to Collaborative Visualization, it is also related to the fields of Scientific Visualisation (SciVis) [68], Information Visualisation (InfoVis) [17] and Visual Analytics [125], all of which are well established with ongoing conferences and journals over the past 20 years. However, in these fields relatively little attention has been paid to the study of collaborative visualisation, especially using advanced interface tools. For example, Isenberg points out that of the nearly 1600 papers published in the three main IEEE visualization conferences from 1990 to 2010, only 34 focused on collaborative visualisation, less than three percent of the total [58]. According to Google Scholar, until now there have been no papers published using the term "Collaborative Immersive Analytics".

This lack of research means there are significant opportunities for research in the field of Collaborative Immersive Analytics. The focus of this chapter highlights these areas and provides some of the background material to help interested researchers. We provide some important background work in collaborative systems, discuss multi-user interaction modalities, highlight lessons learned from collaboration with immersive technologies such as Virtual and Augmented Reality displays and interactive walls and tables, and specifically identify important research topics. We should note that this chapter is not an exhaustive literature 
review, nor a tutorial on collaborative technologies or immersive interfaces in general.

In the next section we begin by introducing a taxonomy of collaborative systems based on space and time, and examples of collaborative immersive technologies that fit into this taxonomy. Then, we discuss the different roles that people have in collaborative systems and how they can interact with the datasets. Finally, we provide a high-level overview of some of the possible research opportunities that could be explored in the future.

\subsection{Collaboration Over Space and Time}

Using Immersive Analytics technology there are many different types of collaboration possible. For example, analysts can work together face to face in a CAVE [82] or a multi-display environment [124], come together from remote locations into the same shared VR environment [77], or leave annotations in datasets to be viewed at different times [35]. Collaborative scenarios like these can be classified according to where they occur in space (distributed vs. co-located) and time (synchronous vs. asynchronous) [65] (see Figure 4). For example, people working together in a lab space are in a Synchronous/Co-located configuration, while those exchanging email over time are working in a Distributed/Asynchronous configuration.

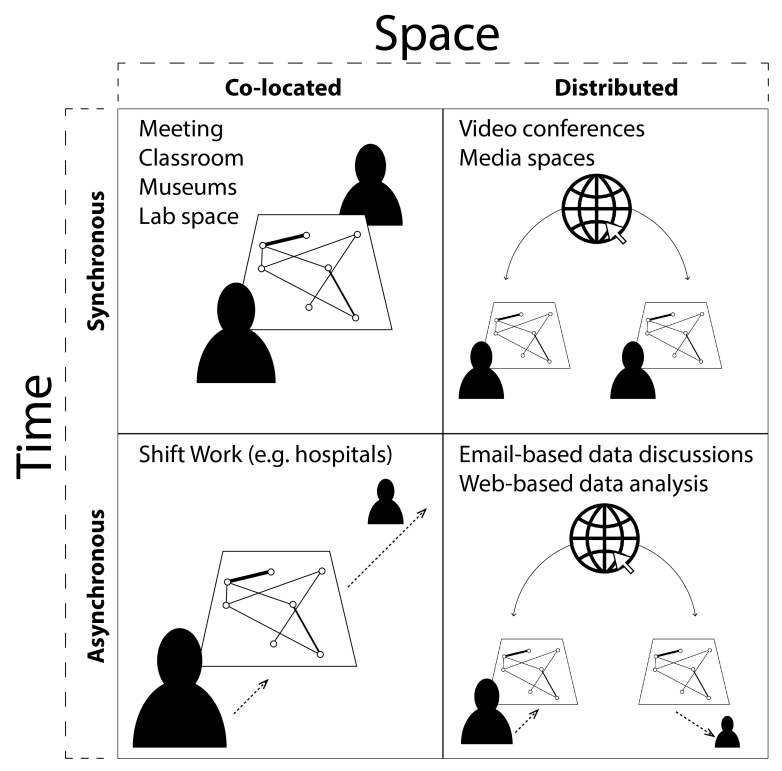

Fig. 4: A space-time taxonomy of collaborative visualisation, from [58]. 
In this section we describe this classification in more detail and review several examples of CIA systems at different points in the space/time collaborative matrix. We also describe some of the lessons that have been learned from previous research that can be used as guidelines for developing collaborative systems.

\subsubsection{Co-Located Synchronous Collaboration}

There are many examples of Immersive Analytics applications where collaborators are in the same physical space and working together at the same time. Marai et al. [82] describes three Immersive Analytics projects where a small group of researchers work face-to-face together inside the CAVE2 environment surrounded by immersive projection screens. In this case researchers brought their own laptop computers into the CAVE2 and were able to work on their private displays as well as use the shared immersive display. Other environments include shared visualizations on interactive tabletops where people stand around the table surface [70] [1], responsive wall displays that multiple people can interact with at the same time [23] [29] [2] [5], and face to face collaborative AR solutions [109] [12]. Figure 5 shows a typical use of interactive tables and walls to support face-to-face collaboration.
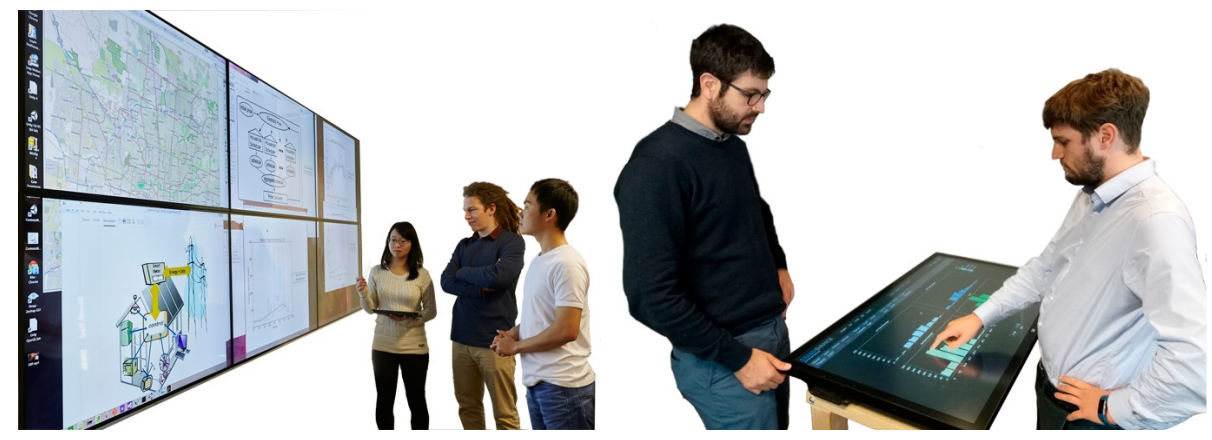

Fig. 5: Examples of using interactive wall displays and tabletops for co-located synchronous collaborative visualization.

Co-located synchronous collaboration has a number of advantages. Collaborators can directly see one another and work together at the same time and so changes they make to the data set can be easily seen by their co-workers [115]. This makes it very easy to have awareness of others which can improve communication, and to move between focusing on their individual work and group work. Collaborators can also easily bring their own externalisation tools (such as computers and notes) into the meeting and share them amongst each other, which helps establish a common ground.

The different immersive technologies available for face-to-face collaboration may also provide unique benefits. For example, with a co-located Augmented 
Reality interface each user has their independent view into the shared dataset that can be customized according to their role [109]. A virtual terrain model could be overlaid with sensor data for an environmental engineer, but an urban designer looking at the same AR model might see traffic information overlaid on the terrain. Similarly, using a tablet at the same time as an interactive wall $[97,140]$ or table [134] allows the user to have their own custom private view and input into the shared display space.

\subsubsection{Distributed Synchronous Collaboration}

Many collaborative analytics tasks are performed by distributed teams, so there can be a need for Collaborative Immersive Analytics applications that support remote synchronous collaboration. For example immersive virtual reality displays can be used to allow analysts working at different locations to come together in the same virtual space to jointly explore complex datasets. Donalek et al. [31] describes how the OpenSim framework was used to create an immersive collaborative visualization space that could be explored in VR head mounted displays (Figure 6). Similarly, high-speed networking can be used to bring remote collaborators into an environment with interactive walls or tabletop displays. For example, the Hugin framework [70] allows the creation of visualization systems where a group of people around an interactive table at one location can connect and collaborate with users around a similar table at a remote location. Other systems have explored the use of mobile devices [88], web browsers [5], CAVEs [66] and Augmented Reality [138] for supporting remote collaboration for information visualization.

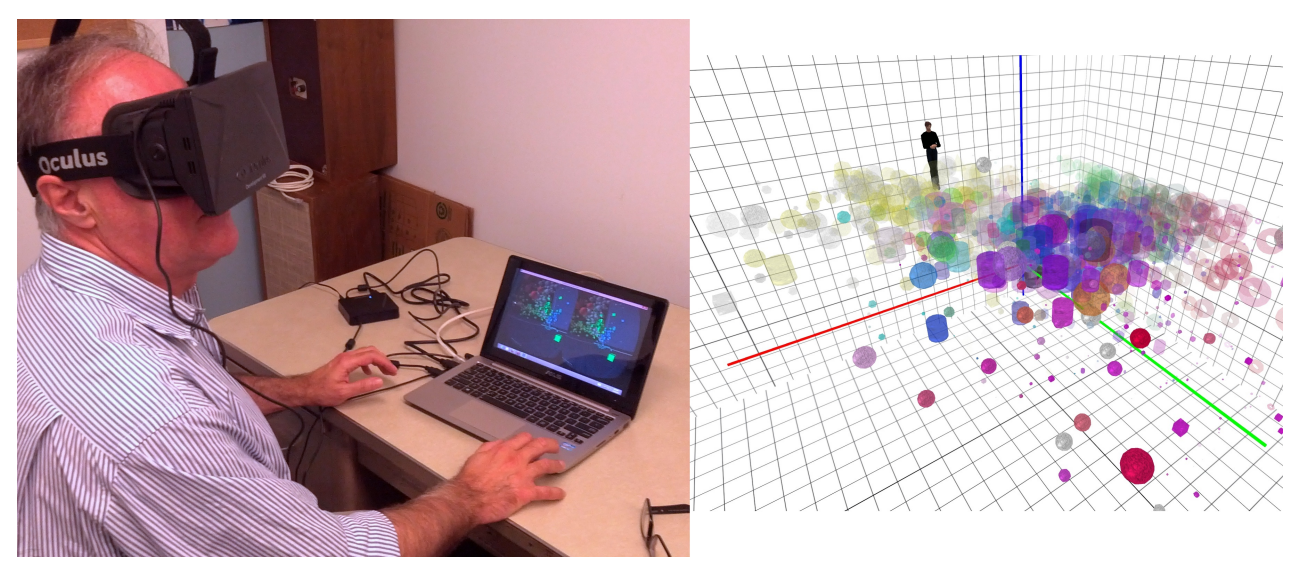

Fig. 6: Using VR to support remote collaborative data visualization, from [31]. User in VR HMD interacting with the system (b) VR view showing remote collaborator as virtual person. 
The main advantage of synchronous remote collaboration tools is that they allow remote individuals to connect and collaborate together. In some cases this can produce a similar performance as face-to-face collaboration. For example, in a study comparing performance on a collaborative visual analytics task in a CAVE (face-to-face) or VR HMDs (remote), researchers found that searching in the HMD condition was as accurate as in the CAVE, and was completed faster [28].

However, there are significant challenges that need to be addressed around awareness and representation of each of the collaborators. For example, traditional video conferencing does not produce the same conversation style as face-to-face interaction [47]. This is because video conferencing cannot adequately transmit the rich non-verbal signals so vital in face-to-face communication and this introducing a communication seam between the participants [61]. In interactive walls and tables, the presence of remote collaborators are often reduced to a pointer icon [43], or virtual shadow of a hand [122]. Collaborative virtual environments immerse users in the same virtual space, but even here the remote participants may be reduced to simple video textures [46] or avatars that cannot convey subtle body motions [31].

Gutwin and Greenberg point out that designing for collaborative systems is difficult because of having to support two goals; designing for individual control over the application and designing for group awareness [43]. They say that collaboration in remote groupware tools is different from co-located collaboration for the following reasons: Groupware systems show far less of the workspace than what can be seen in a physical environment. Manipulation techniques in virtual workspaces are not bound by the physical constraints that exist in physical workspaces. Virtual workspaces can represent and display artifacts in more ways than physical workspaces allow.

\subsubsection{Distributed Asynchronous Collaboration}

For Immersive Analytics most collaborative applications involve synchronous collaboration. Viégas and Wattenberg point out that in general "synchronous collaborative scenarios have been much more widely explored than asynchronous visualization based communication" [131]. However, distributed asynchronous collaboration involves capturing input from people at different times and different places and so can provide some unique benefits. For example, Benbunan-Fich et al. found that asynchronous collaboration can produce broader discussions and more complete reports from group discussions than their face-to-face counterparts [9]. Other benefits include enabling people to contribute whenever they have time to provide input [104], they can work on the part of the problem that they feel most qualified to address [129], and can combine information from a variety of sources [52].

Some efforts have been made to add support for asynchronous collaboration to CAVE and immersive Virtual Reality experiences, mostly through simple recording and playing back of messages. For example, Imai's V-Mail system allows people to send and view asynchronous messages in VR [54]. In this case the user can record a voice message along with their virtual avatar body movements and 
gestures for later playback. Later, this was extended to include a VR-annotator tool for attaching 3D recordings to objects, and a VR-vcr streaming recorder for recording all actions occurring in a collaborative session [55]. Similarly, the Virtue immersive visualization environment provided support for multimedia annotations that allowed users to mark temporal and spatial points for later replay and sharing [111]. More recently, the vAcademia Virtual World shows how 3D recording can be used for asynchronous collaboration in desktop VR environments [89].

There are other examples of non-immersive asynchronous Analytics interfaces that can provide valuable lessons for extending this work. Zimmer and Kerren introduced OnGraX [143], a collaborative synchronous and asynchronous web based tool to support visual analysis of networks. Willet et al. have developed CommentSpace, a collaborative visual analysis tool that allows analysts to annotate information visualisations [136]. The tool allows analysts to add comments or view tags to the visualization over time and link the tags together to aid understanding of the document. In this way Collaborators gain the benefit of seeing the tagged classifications that people are applying to the text, and the semantic links being created. Similarly, Chen et al. [22] developed ManyInsights, a web-based tool for asynchronous collaborative analysis of multidimensional data (see Figure 7). Using this tool people can record their own insights from data, and read the insights of others over time. They found in an evaluation that this led to the generation of more shared insights, and being able to group insights by data similarity was a particularly powerful way to understand the data.

Heer and Agrawala [48] provide an excellent description of design characteristics for asynchronous collaborative visual analytics interfaces, drawing on web-based interfaces. In particular they list design considerations that should be taken into account, such as the value of supporting freeform annotations, using visualization bookmarks, linking to specific views, and clearly showing past actions that have taken place, among others. They designed the sense.us web application for social visual data analysis, showing how these design guidelines can be used [49]. In this case the interface supports view sharing, doubly linked discussions, graphical annotation, collecting and linking of views, awareness and social navigation, and unobtrusive collaboration. Figure 8 shows the sense.us interface and collaboration features.

Viegas and Wattenberg highlight the research opportunities available in the Distributed Asynchronous Collaboration Space [131]. They say "By not fully exploring asynchronous communication of visualization discoveries and processes, the research community is missing an opportunity to make important contributions to visualization research". In particular, they identify three features that are important; (1) Playback: Allowing users to edit a visualization session, picking out only a few key frames, and sharing it with someone else who can rewatch it. (2) Annotation: Enabling users to point to objects and add information. Tracking changes in annotations over time, and supporting group creation. (3) Information Foraging: Supporting how users spread their attention over the 


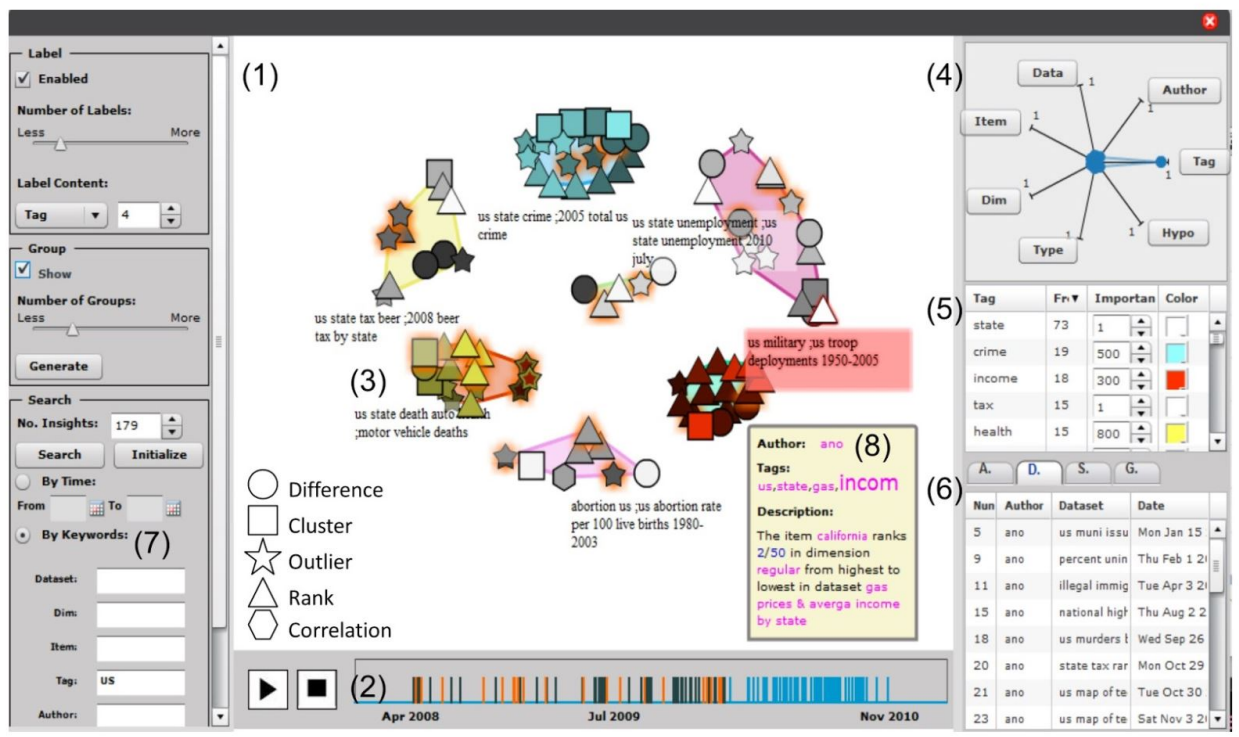

Fig. 7: ManyInsights User Interface [22].

data space, and encouraging users to look at little viewed parts of the dataset. We discuss more about the research opportunities in Distributed Asynchronous Collaboration in Section 8.6.

\subsubsection{Co-Located Asynchronous Collaboration}

Another area which has not been well studied for information visualization is co-located asynchronous collaboration. One common example of an asynchronous collaboration tool is a shared public display that people can view at different times. For example, Carter et al. [19] describe a public display interface that they developed for showing common topics of interest among emails and the current locations of the email writers. This display was designed to encourage more collaboration between co-workers. Other examples of using collaborative public displays include Groupcast [84], CWall [114], and the Notification Collage [40]. Figure 9 shows the Notification Collage interface with a variety of different media items (web pages, notes, video feeds, desktop views, etc.) posted on a shared public display.

The availability of public displays has prompted researchers to experiment with asynchronous, co-located visualizations, for example, in the form of ambient information displays [113]. In this case, public displays in the physical environment are used for information visualization. Pousman and Stasko [100] provide a good overview about how such systems can be used for casual information visualization in a public setting, such as showing bus timetable information, social networks, 


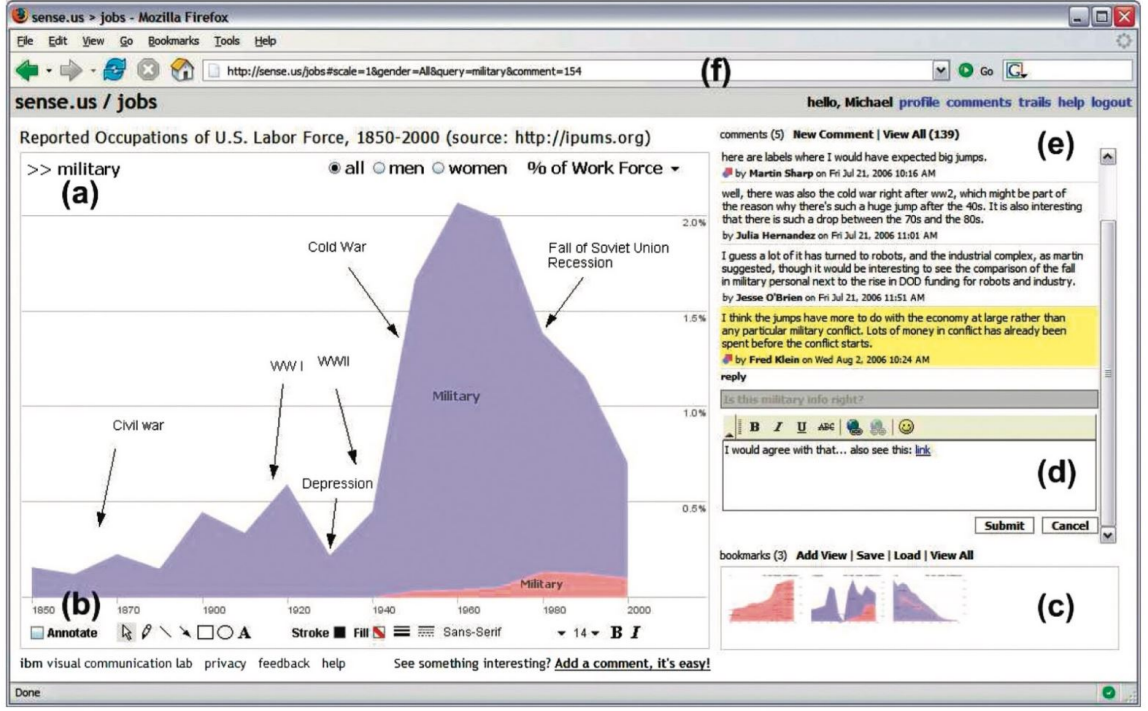

Fig. 8: Sense.us interface [49]; (a) an interactive visualization applet, (b) annotation tools, (c) a bookmark trail of saved views, (d) text-entry field for adding comments, (e) threaded comments attached to the current view, (f) URL for the current state of the application, updated automatically as the state changes.

or photo collections. Similarly, Vogel provides an overview and design guidelines for interacting with co-located displays [133].

Co-located asynchronous collaborative systems have many of the benefits of distributed systems, with the additional benefit of collaborators viewing the same physical space. Viegas and Wattenberg [131] mention that this offers some interesting design opportunities for the physical surroundings around the visualization, such as providing pens and paper for people to add their own notes around the display. Heer and Agrawala [48] point out the value of collaborators being able to see the same collaboration space, and other researchers have identified the value of having externalization tools that help people create and add their own insights [81]. Overall, having a common physical reference space with shared visualization and external objects should significantly help collaborators achieve common ground and shared contextual understanding [25].

\subsubsection{Mixed-Presence Collaboration}

So far we have talked about collaborative experiences that fit into only one quadrant of the time and space taxonomy. However, it is also possible to create experiences that link together two or more quadrants. For example, Mixed Presence Groupware (MPG) systems are collaborative systems that connect both co-located and distributed collaborators [53] [120]. As such Mixed-Presence 


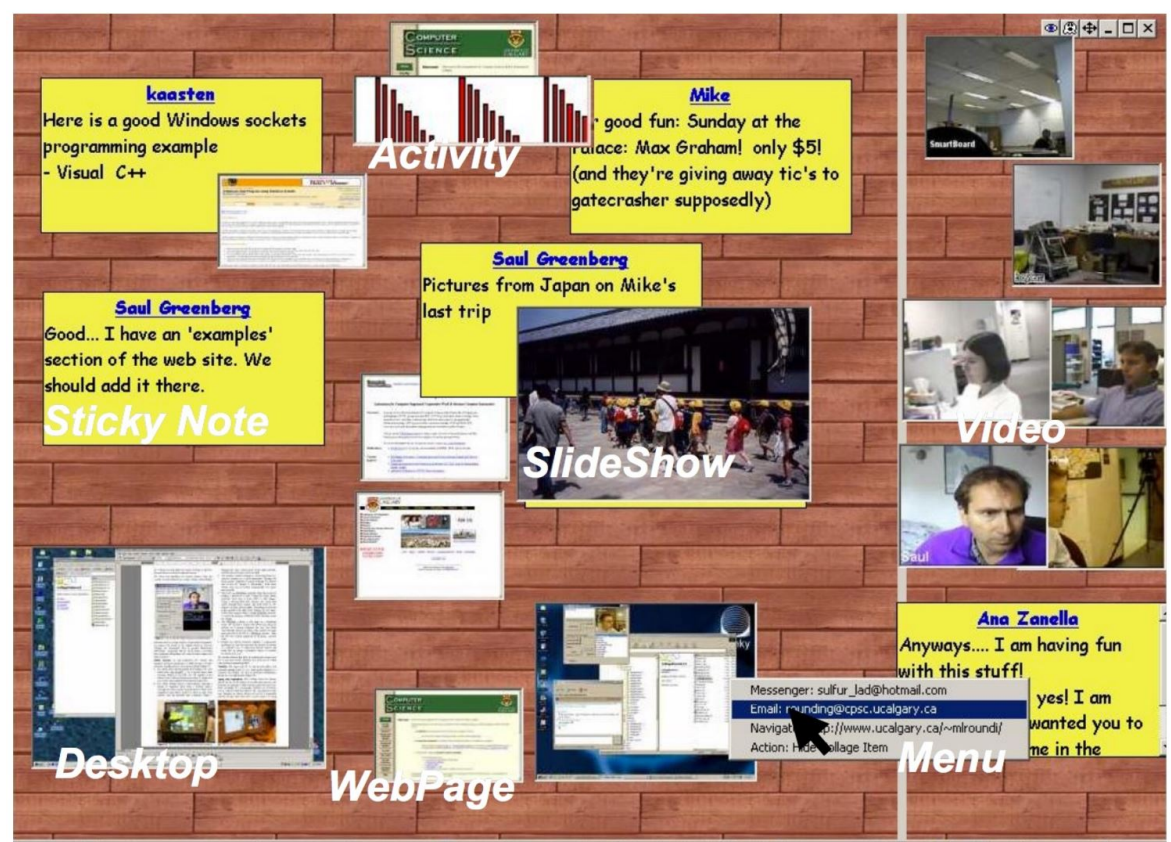

Fig. 9: The Notification Collage interface [40].

systems support synchronous collaboration between people in the same location and a remote location (see Figure 10). For example, the NICE Project supported collaboration between groups in one immersive CAVE with remote collaborators in other VR environments in an educational setting [67]. Other examples include interactive tables that include local and remote participants [106], and combining a VR conferencing space with a physical interactive table [103]. Figure 11 shows a typical mixed presence tabletop application where remote users are represented by video shadows of their arms (from [Tuddenham 2007]).

MPG systems combine the advantages of co-located and distributed synchronous collaborative interfaces, allowing distributed groups of people to work together. However there are a number of design challenges that need to be addressed. For example it can be challenging to enable co-located and remote users to have the same level of mutual awareness, and there is a need to provide some representation of the remote users into the local users space [98]. For example, video arms [121], as seen in Figure 11, can show where the remote users are reaching into the shared interactive space. It is also difficult to enable users to share notes, their own device screen and other local physical artifacts with remote users. Robinson and Tuddenham provides a list of design guidelines to address some of these challenges [106]. 


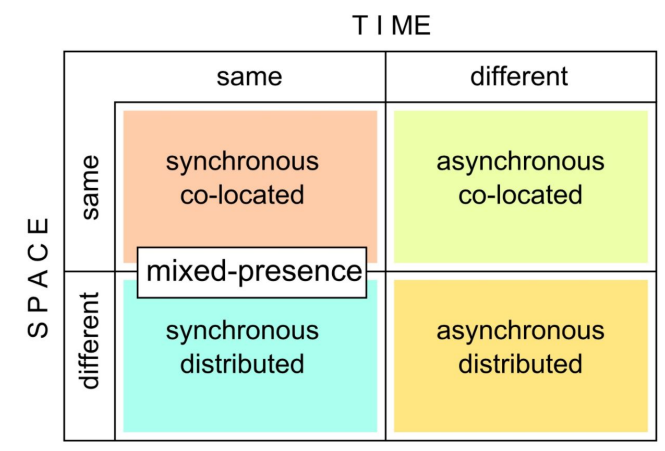

Fig. 10: Mixed Presence Groupware systems combining co-located and remote groups.
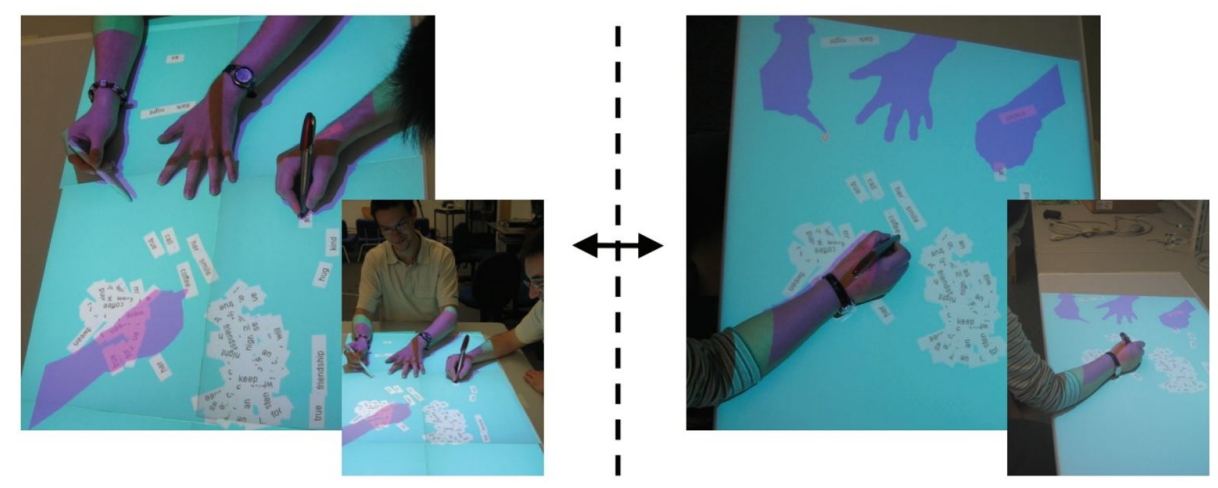

Fig. 11: Mixed Presence Tabletop System [106].

\subsubsection{Lessons Learned}

A number of lessons have been learned from this research. From the co-located synchronous research (e. g. [82]) the following features were found to be important:

- Supporting different independent viewpoints

- Enabling the use of different tools for different data

- Supporting face-to-face group work

- Using different data representations

Churchill et al. [24] reviewed a wide range of collaborative settings and state that the following should be provided in order to support good group communication and problem solving:

- Shared context - the shared knowledge and context around the data

- Awareness of others - users being aware of others' actions 
- Negotiation and communication - people being able to freely talk with each other

- Flexible and multiple viewpoints - showing different viewpoints depending on roles

As can be seen from the reviewed systems, there are a wide range of different ways to achieve these requirements. In addition, previous research has shown that groups need to be able to have access to externalization tools, such as notes or laptop computers that enable them to record their insights and organize the results of their analysis [80] [48]. It is also important to enable collaborators to have access to both individual and shared workspaces [43] [60], and to enable them to easily shift their focus from their own work to the work of their collaborators [32]. Marai et al. point out that people from different backgrounds want to see data represented in different ways, so there should be multiple representations available and a variety of tools for interacting with the data [82].

In this section we have categorized collaborative systems according to how they support collaboration through space and time. Another important element of these systems are the various roles that the users have, and how the technology can support these roles. In the next section we describe the different roles used in CIA systems.

\subsection{Types of Participants in CIA}

In collaborative systems participants may have different roles or types of engagements with the system. For example, during a data driven presentation there are typically one or more people presenting while other constituents are audience members that generally do not interact directly with the presentation material. The roles of the people in the collaborative setting define the level of engagement that they have. Isenberg et al. [58] discuss how there are three different levels of engagement with collaborative visualization systems:

- Viewing: people are consuming a data presentation without interacting with the data, such as in a lecture presentation.

- Interacting/exploring: people have the means to choose alternate views or explore the data.

- Sharing/creating: people are able to create and distribute new datasets and visualizations to be explored.

Zhu [142] discusses role-based collaboration and points out that for efficient collaboration there needs to be support for asymmetric expertise and authority. For example, in a meeting the collaborative system needs to allow a presenter to control the image presentation, while the images shown should be made visible to all of the viewers in the audience. Similar to theater acting, storytelling can be enhanced by adjusting your presentation to suite your audience and react to their response. Sole [116] points out that effective knowledge-sharing requires storytellers to streamline their narrative by removing (or temporarily hiding) 
preparatory parts of the analysis that may overwhelm or confuse the audience. Sole asserts that the other most essential part of a good narrative is to provide a surrogate experience for the recipient. Immersive Analytics uniquely offers a sensorial advantage for enveloping recipients in a story thereby increasing the effect of Suspension of Disbelief. For example, analysts within a CIA willingly interact with abstract visualizations of data that may not realistically represent the original source of that data.

Collaborative Immersive Analytics builds upon these archetypal roles, but also enables the ability to mix roles. Given that CIAs are necessarily more highly mediated and interactive than traditional visual analytics platforms, it may be quite natural for participants to switch back and forth between passive viewer and active explorer. In fact, Heer et al. [49] discuss how their sense.us platform was a social space for information visualization designed to facilitate data driven discussion and debate. CIA offers a unique opportunity for example in Command and Control scenarios where users may spend most of their time monitoring data feeds and then switch contexts into active analyst and explorer roles as problems arise. It is thus important that data representations and interfaces of CIAs can support fluid transitions between these roles. Another consideration when designing Collaborative Immersive Analytics is the number of creators and consumers. Here are some of the most common formats for collaborative analytics:

- One to self (e.g. data discovery that might be shared later)

- One to some (e.g. reporting findings to colleagues)

- One to many (e.g. presenting reports to stakeholders)

- Many to many (e.g. department heads presenting at employee town halls)

- Many to one (e.g. department heads presenting to CEO)

- Machine to one, some or many (e.g. AI returning results to analyst)

Up until about 10 years ago, the predominant method for analysis was through a guided analytics approach whereby data scientists created visualizations that were presented to and utilized by other users. In the Business Intelligence industry, this meant a very small group of individuals produced highly structured dashboards and reports which were consumed by specific departments within large organizations. Over the last 10 years though, there has been a massive shift to what is referred to as Self-Service Analytics in which users now have the ability to input, access, and analyze data directly by themselves. This transformation has enabled a new form of data discovery which allows for more people to ask more questions and solve more problems. This trend towards creating new collaborative analytics platforms is nicely articulated by the ManyEyes project [132] mentioned earlier. ManyEyes attempted to "democratize" visualization by not only serving as a discovery tool for anyone with data, but also as a platform to prompt discussions around data for large groups of users. This example best typifies the "many to many" form of collaboration.

Machine learning and artificial intelligence (AI) are being applied more and more towards analytics. There are many advanced analytics systems which employ 
AI to automate various aspects of the analysis process including everything from data cleansing, preparation and modeling, to dynamically creating visualizations or data-driven narratives through natural language generation, to alerting users of "unusual activity" and predicting future trends. This new "analytics agent" can be considered as a third axis in a collaborative user matrix in Figure 12. Within a CIA, this collaboration with the AI could be represented through a friendly $3 \mathrm{D}$ avatar such as those we already interact with aurally through automated voice systems. However, ultimately AI systems will likely mature sufficiently so that they will replace some human knowledge workers through new forms of deep learning combined with expert systems.

This possible introduction of machine intelligence in the analysis process complicates further the design of CIA, as it adds a new level of complexity in the form of an additional actor or role (beyond the existing human ones), whose actions need to be made aware and interpreted by the human collaborators.

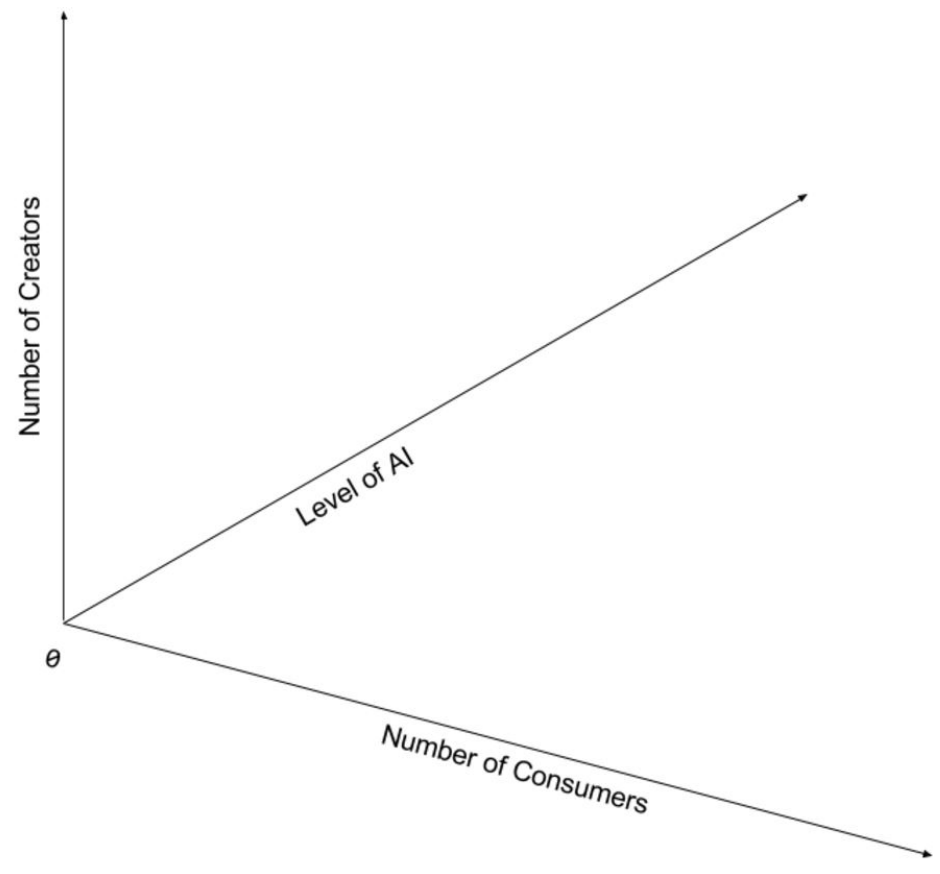

Fig. 12: Collaborative User Matrix.

Given that CIAs can offer more interactivity and potential for greater contribution by more participants, it can also encourage dialogue and debate around data analysis. If a chart is presented in 3D and one can easily move around it and interrogate it, will that simple shift in perspective also transform users into active 
listeners? Or can we go beyond simply allowing for the ability of multiple users with various roles within an Immersive Analytics environment to also enable them to have suitable means to effectively collaborate? In the next section we will explore how different types of interaction modalities enable participants within a CIA to engage in various analytic tasks.

\subsection{Interaction in CIA Environments}

Deciding on appropriate interactions to support any visual analysis task is important, as interactions facilitate the dialogue between analysts and their data [126]. When considering collaborative environments in particular, analysts are in dialogue not only with the data, but also with each other, and the choice of interaction technique can influence the nature of collaboration and the awareness of others [32,44]. For example, Prouzeau et al. [102] observed that when analyzing graphs on a wall display, pairs using techniques with a large visual footprint adopted tighter coordination strategies than pairs using techniques that were more visually localized.

Interaction design in Collaborative Immersive Analytics (CIA) environments faces similar challenges to other visual analysis environments [126]. For example they require interactions to support potentially complex analytic tasks, such as defining and filtering unwanted data, requesting new representations of the data, etc. The chapter on interaction covers these extensively (Chapter ??). Thus the interaction vocabulary in such environments needs to be rich enough to go beyond simple actions such as pointing (a task studied in detail in immersive environments). Moreover, collaborative environments must also take into account other challenges and opportunities identified in previous work on collaborative immersive environments more generally.

This section discusses preliminary synchronous interaction (co-located or distributed), as it directly impacts collaboration aspects such as awareness and coordination.

\subsubsection{Synchronous Co-located CIA}

Synchronous co-located CIA happens historically in shared physically large immersive environments such as CAVEs, walls and multi-display environments (MDEs) that surround the viewer, where analysts can move freely around the space (such as the ones seen in Figures 1 and 5). Movement has been linked to benefits such as avoiding occlusion by others in CAVEs [21], and correcting for possible visual distortion in walls [13].

Movement: While collaborative interaction from stationary positions with mice and keyboards is still possible $[16,57]$, it does not leverage the full rendering capacities of these immersive environments. In CAVEs analysts can move inside the data itself and view it from different perspectives. With walls they can see data at different scales, coming close to the display to see details and further back to get an overview [7]. With tables, they can see data from different angles by moving 


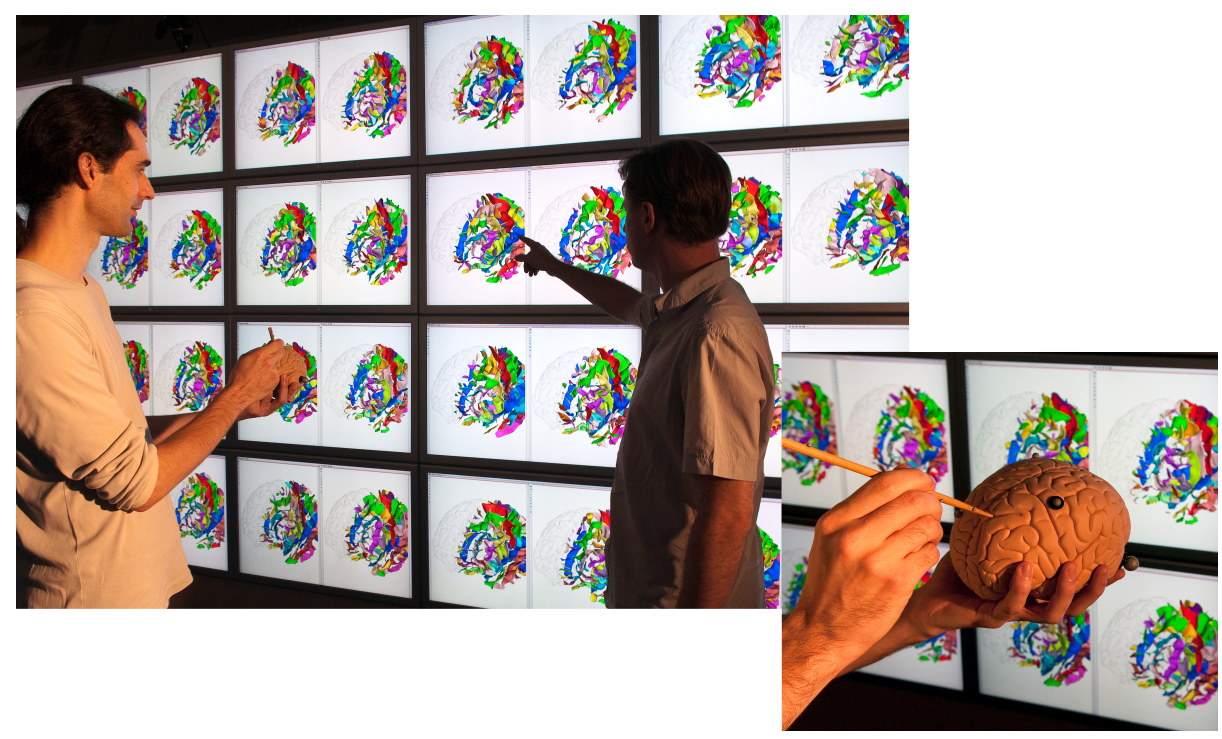

Fig. 13: Interaction using a tangible prop of a brain to rotate brain images, and a stick that defines the camera position (left), and close-up (right) from [39] (used with permission, (CNRS-Phototheque - Cyril FRESILLON).

around the table. In all cases, movement can be seen as an implicit interaction for navigating in the data space. Movement has also been used as an explicit interaction, for example to trigger dynamic changes in content rendering [7], or to invoke actions such as dynamic filtering depending on the analyst's position and movement [63]. While such approaches can increase the interaction vocabulary, they need to be carefully considered in collaborative environments, where implicit actions as simple as moving may cause large scale visual changes that could disturb other analysts. This issue is particularly prominent in large-scale stereoscopic environments (such as CAVEs), where almost always only a single person controls perspective and interaction, even though multiple people can move in the space.

Pointing and Gestures: Existing work has looked at different interaction alternatives that support free movement. Research on CAVEs, walls and multidisplay environments have considered extensively pointing techniques (using hands or dedicated devices) [4,92,93], that combined with on-screen menus and widgets, could cover the complex interaction needs in CIA environments. Pointing actions can be easily seen by colleagues, increasing the awareness of others. Nevertheless, the existence of persistent on-screen menus on the shared collaboration space takes up space that could be used for displaying data, while menus invoked on the fly can be disruptive to collaborators sharing the space.

As an alternative, hand or full-body gestures can activate commands (e.g., [117]) and provide a rich interaction vocabulary without taking up screen space. They provide awareness of the actions of others, given that these gestures are 

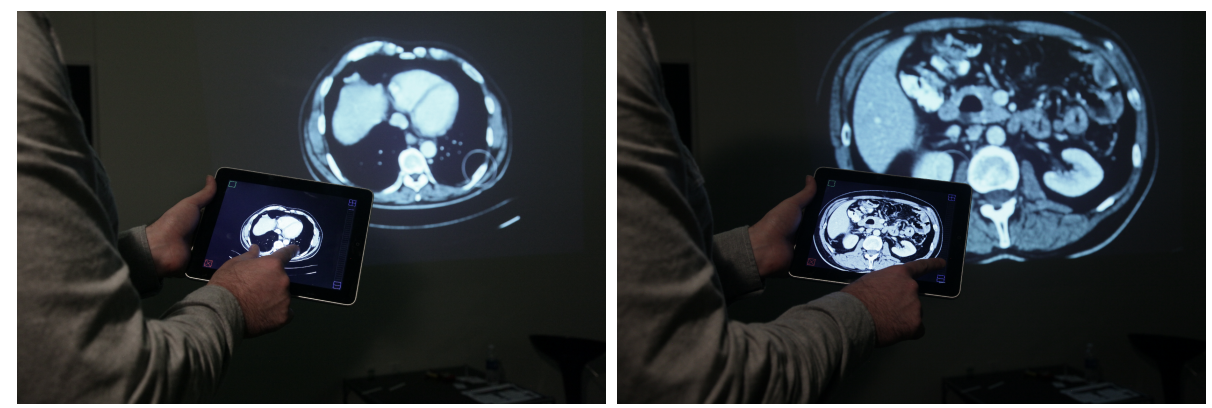

Fig. 14: Multi-touch input on a tablet to zoom and scroll through a stack of brain-scans [97] (used with permission).
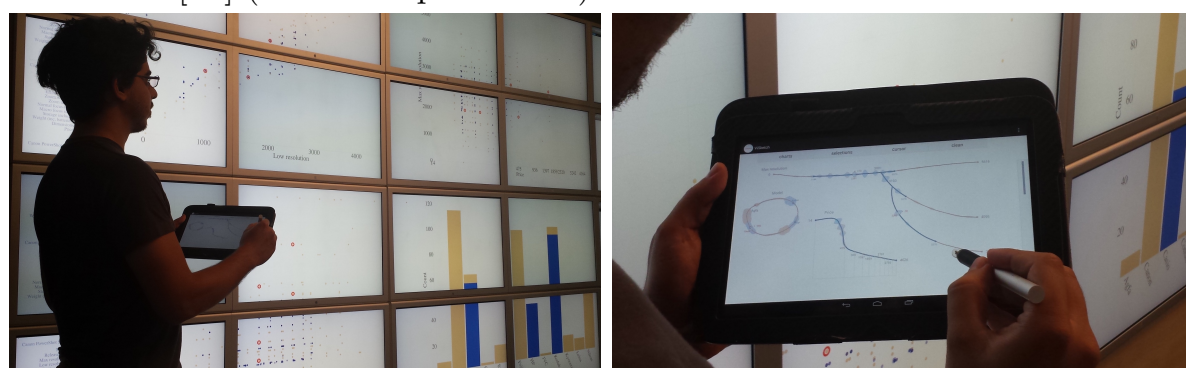

Fig. 15: A user sketching the sliders they need for their exploration [127] (used with permission).

easily seen by colleagues sharing the space. Nevertheless, full-body gestures can prove tiring during long periods of work [139] required by some analytics tasks [126], and gestural vocabularies can be large thus coming with a learning cost $[69]$.

Dedicated Devices: Researchers have also examined the use of personal, dedicated, or generic, mobile devices. For example, in order to analyze brain scans on a wall display, Gjerlufsen et al. [39] suggested using a dedicated tangible prop of a brain to rotate and translate the scans (Figure 13), and physical tools to indicate camera viewing directions; Olwal et al. [97] suggested using dedicated software running on tablets to provide interaction tools and different views of the brain between colleagues in medical teams (Figure 14).

These examples illustrate the large possible range of such mobile devices in terms of both generalizability across analytic tasks, and awareness of others. Interaction with dedicated tangible props (such as a model of a brain), comes with a high degree of awareness since props used by colleagues are easy to identify, but their utility is limited to specific data domains and analysis tasks (exploring brain scans). On the other hand, more general purpose devices can be adapted across analysis tasks and data. For example, devices with multiple degrees of freedom (e.g., [11,37]) allow for a rich input vocabulary while at the same time being general purpose. Existing mobile devices, such as smartphones and tablets, can also be appropriated, adapting their content to provide a personalized interface 


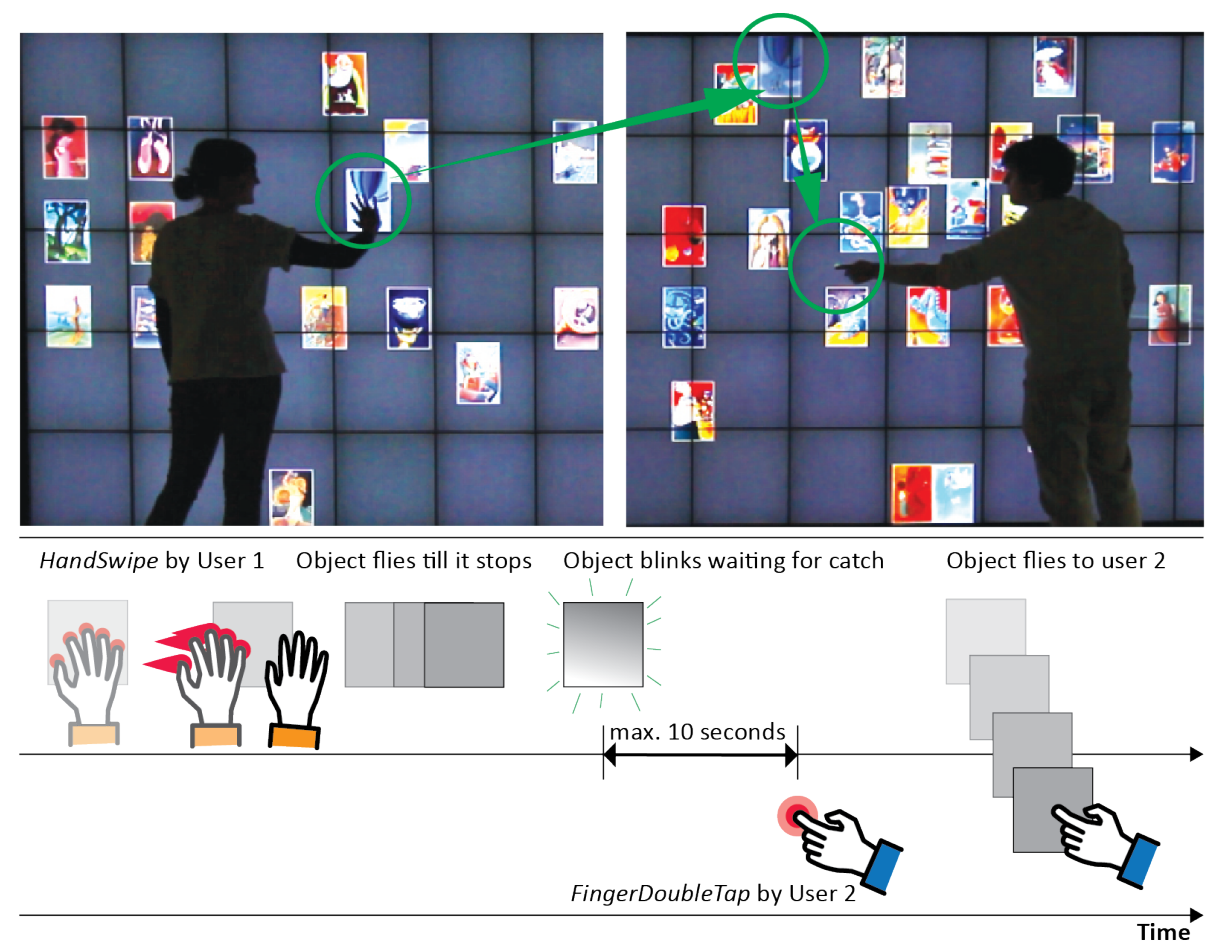

Fig. 16: A user throws an item and another performs a gesture to make it "fly" to their location in CoReach [79] (used with permission).

for analysis tasks or personal views of the data $[97,134,140]$. Mobile devices can also be combined with physical widgets [64] or be customized by analysts, for example allowing them to sketch their own interfaces based on their analysis needs [127] (Figure 15). Because these more general purpose personal devices can be adapted to help analysts perform many tasks, they usually provide limited awareness of the actions of others. In this case, additional awareness information needs to be given during collaboration, for example in the form of colored cursors, or other highlights to indicate the work of others [44]. Mobile devices, dedicated or general purpose, need to be carried around while analysts are moving, which could prove cumbersome in long duration analysis tasks.

Touch and other modalities: Physically large immersive environments often include displays that can act as touch surfaces. In the past researchers have considered direct touch interaction as input in collaborative analytics on very large surfaces such as walls $[62,102]$ or tabletops [60,91]. Direct touch provides immediate awareness information about where others are working in the shared space, and allows for direct manipulation [112]. Nevertheless, touch technology may not be always available, and even if it is, it requires users to be close to the display, missing the benefits of movement discussed before. This 
is where multi-modal interaction can prove useful; for example Liu et al. [79] use a collection of collaboration techniques based on direct touch on a wall and distant interaction using mobiles. More generally, multimodal interaction can prove beneficial for collaborative settings for diverse reasons. For example, adding voice commands on a touch tabletop can enhance the gesture vocabulary and potentially increase awareness of others' actions [128]. Or providing personal devices coupled with the shared interactive surface, allows colleagues to fluidly move between individual and team work [110], and chose when to share their individual analysis results with others [85].

Collaborative actions: Co-located collaborative settings also allow the creation of new collaborative interaction techniques, where users combine their actions in order to create a single more powerful interactions. Morris et al. [90] present actions by multiple colleagues that the system interprets as a single command, in order to pass ownership or trigger global changes for example. Tse et al. [128] allow colleagues to combine verbal commands on a tabletop to group objects. Isenberg and Fisher [59] provide collaborative brushing and linking interactions on tabletop visualizations and Liu et al. [78,79] look at how multiple collaborators can share actions such as moving objects across different areas on a wall display (Figure 16).

\subsubsection{Synchronous Distributed and Mixed Presence CIA}

Synchronous distributed immersive technology, such as HMDs provides analysts with the ability to chose whether to share exactly the same view or completely different ones. As with individual analysis in these environments (see Chapter ??), a rich input vocabulary can be achieved using pointing and virtual menus [15], more elaborate gestures $[6,26,74]$, or even mobile devices [36]. What is unique about this setting is that, contrary to physically large environments where awareness of others often comes from seeing the location and actions of others within a shared physical space, here analysts may be unaware of the focus and view of their colleagues. In order to maintain awareness of others, interaction needs to provide feedback not only to the user taking an action, but also to their colleagues. For example, researchers have proposed the use of avatars to represent their fellow analysts within the virtual world, starting from very abstract representation of others [10] to full 3D body models [101]. Analysts' actions need also be accompanied by feedback presented to their colleagues, for example in the form of highlights of their area of focus [38] that can be very fine grained (e. g., through eye-tracking [42]), and the ability to share views with others [96].

Given the introduction of augmented reality technology, it is possible to have scenarios where analysts are physically co-located, but have personal views of their data superimposed on the virtual environment. In these mixed reality environments, most interactions that are possible in physically large environments are possible here as well (with the exception possibly of touch interactions on shared surfaces). Nevertheless, in this context, awareness of actions can prove problematic. Even if the physical position of analysts and their physical actions (e.g., gestures) are visible, there is no guarantee that they are transparent to 
their colleagues since they are not sharing a common view. This problem is similar to the awareness challenges present in distributed environments. It may even be more pronounced in mixed reality settings: in distributed situations, representations of colleagues can be artificially placed close to their areas of focus; in a mixed reality setting, we have a strong visual presence of colleagues moving inside an analyst's field of vision (but not necessarily sharing a common view), that may be hard to override in order to represent their virtual focus.

Designing interaction for situations where some colleagues are co-located and some distributed, i.e., mixed-presence collaboration, presents possibly the biggest challenge. The analysis environment needs to provide awareness support for the actions of co-located colleagues, while at the same time providing representations of the distributed partners and/or their actions. All this, in an environment that is likely already occupied by large amount of visualized data.

\subsubsection{Asynchronous CIA}

So far, we have considered only synchronous interaction in collaborative environments. Nevertheless, analysis tasks can be long, requiring several work sessions [126], or analysts may work individually but share their analysis later on. These asynchronous analysis sessions can be done either distributed, or even colocated (working in shifts). In these case, some interaction challenges are similar to single user interaction. Nevertheless, there are new problems that arise when analysts work asynchronously, such as understanding where their colleagues left-off with their analysis, where they focused on, etc. More general, supporting hand-off in asynchronous analysis [141], both co-located and distributed, becomes crucial. Here, we require techniques that leave a trace of the analysis work and progress of colleagues. These can be explicit, for example annotations or summaries left behind for others [94,105]; implicit, such as summaries of all past interactions (as is done in [14]); or a combination of both, where the analysis system can help the analyst to tell a story $[18,34,75,107]$ of their work to bring their colleagues up-to-speed (see Chapter ??).

\subsection{Opportunities for Research}

Collaborative Immersive Analytics is an emerging research field and it is still underexplored. As it is challenging to identify a pertinent path to explore in CIA, this section aims to provide a non-exclusive list of important research questions to guide research in this new domain. Previous work has identified important characteristics of Collaborative Visual Analytics. Heer [48] identified the following topics to explore:

- Division and allocation of work

- Common ground and awareness

- Reference and deixis ${ }^{1}$

\footnotetext{
${ }^{1}$ Deixis refers to words and phrases, such as "me" or "here", that cannot be fully
} understood without additional contextual information. Source: Wikipedia. 
- Incentives and engagement

- Identity, trust, and reputation

- Group dynamics

- Consensus and decision making

Virtual/Augmented/Mixed reality, wall-size displays and tabletops can lead to more immersion and presence. The nature and affordances of such immersive environments may have an influence on how a group of users work together. This chapter presents a set of questions related to the space/time matrix and important topics previously identified in Collaborative Visual Analytics, with respect to immersive environments.

\subsubsection{Virtual reality for Immersive Collaborative Analytics}

CAVE-style setups and large display or multi-display environments are a natural space for collaboration. CAVE-style setups are rooms with screens surrounding the users. Collaborators view and talk to each other, and can, for example have face-to-face discussions. Collaboration in Virtual Reality can also be alternatively supported by networked head-mounted displays, or fishtanks. Cordeil et al. [28] found that performances were comparable between CAVE-style displays (Figure 17) and HMDs (Figure 18), but raised some usability issues for collaboration. However, there are other Virtual Reality displays that need to be compared for collaboration. Hence it is important to conduct research with different display form factors.

\subsubsection{HMDs and face-to-face communication}

While HMDs offer an alternative to wall displays and other CAVE-style environments, they obscure the face of collaborators. This can potentially be a major impairment as facial expressions are used as visual cues for different reasons, including establishing a common ground or assessing and discussing findings [48]. The study of Cordeil et al. [28] did not reveal a major issue with this impairment but they tested only low-level graph visualization tasks and did not test analytical reasoning. In this condition, using only conversation and gaze was probably sufficient to complete the tasks. An interesting opportunity for research is to measure the effect of not seeing the face expressions of collaborators:

- When they are sharing insights: how does it affect engagement?

- On the group dynamics: how does it affect leadership?

\subsubsection{CIA and scale: number of users}

Research has often studied collaborative tasks with head-mounted displays or shared large displays, involving only two collaborators. In real-life applications, collaboration potentially involves larger team sizes. There is a compelling case to study the impact of using both HMDs and shared displays, such as walls and tabletops, with large groups in terms of situation awareness. In order to design 
and develop future techniques, systems and platforms that support immersive and collaborative analytics, it is crucial to study how different group sizes collaborate with visualizations. A possible (non-restricted) list of research questions related to group size, could be:

- How do collaborators locate each other's positions in collaborative virtual environments?

- How do collaborators share insights with others (either in virtual or collocated environments)?

- How do collaborators share/find each other's cues?

\subsubsection{Distant Collaborative Immersive Analytics}

Little research has been focused on remote collaboration using immersive display technologies. Some studies have been carried out in the scientific visualization domain [31] and the robotics domain [73]. Those studies often investigate the ability of users to perform their tasks in such environments, and often use artificial or abstract tasks such as puzzle solving. However, collaborative analytics requires a higher level of engagement from collaborators when analyzing and understanding data. The study of engagement of team members in remote, immersive and collaborative data analytics remains widely unexplored. To this regard, the following research questions should be investigated:

- Does immersive and collaborative analytics bridge the distance gap in remote collaboration scenarios?

- What is the impact of immersion in collaborative analytics quality?

- What is the effect of immersion on remote collaboration: engagement, group dynamics?

\subsubsection{The design space of Immersive and Collaborative Analytics}

Immersive display technology is very heterogeneous. It includes head-mounted displays, CAVE-style environments, wall-sized displays, tabletops, and multidisplay environments. Those displays also vary widely in size, resolution, and modality of interaction. Choosing which technology is more appropriate for the given tasks and needs of analysts, and designing for them, is a challenge. There is a great opportunity for research to define the design space of collaborative immersive analytics in order to guide the design of immersive collaborative platforms. Moreover, we can envision situations where a mix of these technologies can come in play, for example a group of analysts working in a CAVE and a colleague connecting remotely using a HMD. Understanding how collaborative analysis is affected by analysts using asymmetric technology (in terms of awareness, deixis, group dynamics), and how to design for it, is an important research direction.

\subsubsection{Asynchronous collaboration}

Collaboration can occur asynchronously for various reasons: collaborators work in shifts, in different time zones, they have different schedules, etc. In this situation, 
users view, analyze and understand data at different moments in time. We can identify two roles:

- A "hand over" role: users who have finished working with the visualizations

- A "take over" role: users who come after the previous group to keep working with the data visualization.

It is important to understand what the users' needs in those two roles are and how immersion can be used to better support hand-overs.

On one end, immersive technology can help preparing high fidelity handovers to communicate the results of data analysis. There are various ways to create a handover for the next users. For example, motion-tracking technology can be used to record precise hand gestures and interactions with the visualizations, and stereo cameras can record a user's body and face. Such recording can be used to facilitate telling a story (Chapter ??) about the work done with the visualizations. However, telling a story is only one form of asynchronous collaboration handover. A group of users may require the help and draw the attention of another user or a group of users to examine a specific issue. On the other end, a user who enters a collaborative space needs to be updated with the situation. The tasks of this user can be to consult the handover, resolve issues, answer questions, provide input for a specific problem, etc. In the context of Collaborative Immersive Analytics, it is unclear which techniques are better suited for specific scenarios. Hence, there is a research opportunity to study how to convey the state of progress of collaborative visualization in immersive and asynchronous collaborative analytics.

\subsubsection{Channels for collaboration}

Collaboration between members of a team is supported by various channels of communication. Those channels include (but are not limited to) oral communication, gaze direction, deixis (e.g. pointing) or feedback from other members' actions. Since CIA can simultaneously occur across different types of immersive technology, it seems important to evaluate, according to the users' tasks, which are the essential channels to support efficient group work.

\subsubsection{Evaluation of CIA systems}

The evaluation of CIA systems, as with all collaborative visual analysis systems is a challenging matter, as it needs to consider both task-related and teamwork related aspects [56]. Because collaborative environments incorporate a large number of variables to consider, they are often evaluated through field and laboratory observation studies [56]. The variables to consider increase in immersive environments, as colleagues may have asymmetric collaboration, be joining the work with different immersive technology, making evaluation even more challenging. Recent years, have also seen an increase in controlled experiment evaluations in collaborative immersive environments (e.g, [79], [102], [20], [78]), where researchers try to tease out effects of particular factors, including immersive technology. For example, Cordeil et al. [28] compared CAVE environments to Head-mounted displays in a graph analysis task (Fig. 18,17). Clearly, it is a mix of 


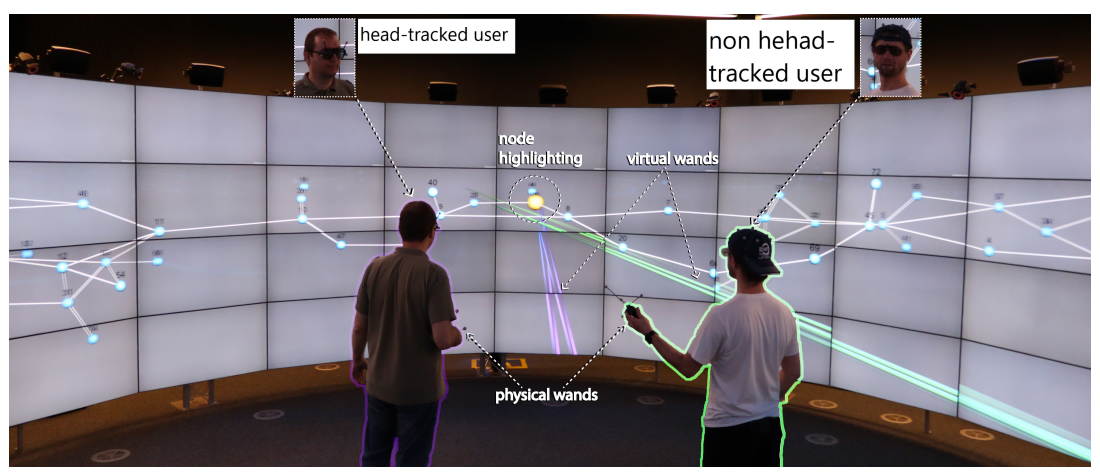

Fig. 17: Two users visualize a 3D network of abstract data in the CAVE2. The $3 \mathrm{D}$ immersive visualization can only be presented from the user wearing the head-tracker (left) [28] (used with permission).

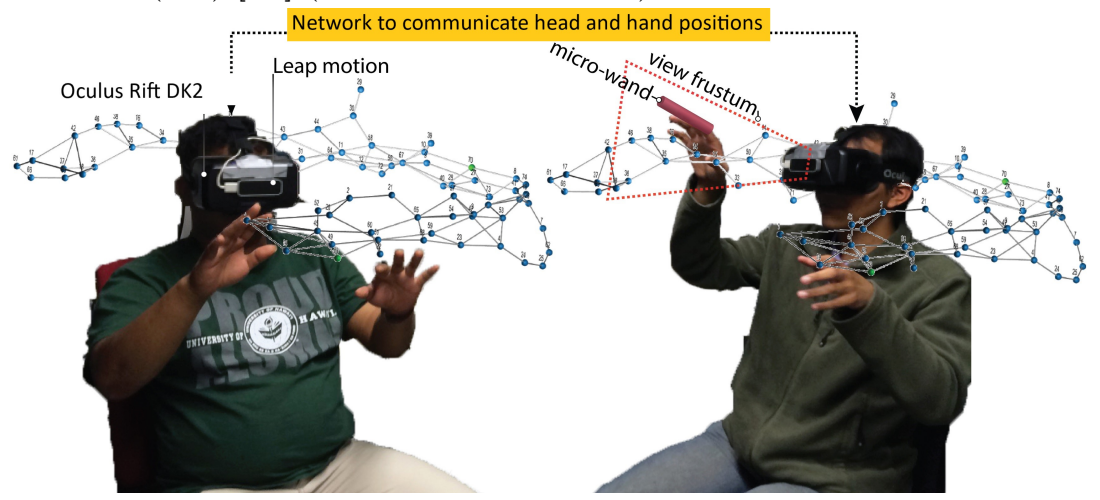

Fig. 18: Two users visualize a 3D network of abstract data in a network application with head-mounted displays. This platform allows independent head-tracking. Gaze cues and interactions are communicated on the network [28].

both open-ended and controlled evaluations that can provide practitioners with holistic insights. Hence, it is important to build evaluation frameworks for CIA, based on subjective, objective and collective measures to quantify the efficiency of collaboration. Such frameworks can certainly be built upon existing ones, for example ones coming from social presence [41].

\subsection{Conclusion}

This chapter introduced the concept of Collaborative Immersive Analytics (CIA), defined as the shared use of new immersive interaction and display technologies by more than one person, to support collaborative analytical reasoning and decision making. As shown in the introduction, CIA is at the intersection of the fields of Mixed Reality, Visual Analytics and CSCW and as such there are many technologies and approaches available for building CIA systems. These 
were classified according to a space-time taxonomy, and examples of systems in each quadrantof the taxonomy were provided.

From these examples we see some of the benefits of CIA systems, including enabling teams to work together, using emerging immersive technologies for intuitive data explorations, supporting multiple devices and tools, and seeing multiple representations of data. Perhaps most importantly CIA systems have the potential to enable teams to overcome the barriers of distance and time to collaborate together in the most effective way possible.

To fully realize this potential CIA systems must be carefully designed. The chapter covers the different roles collaborators have in such systems, such as viewing, interacting and sharing which should be accommodated in the system development. It also reviews the types of input modalities that could be used to design intuitive interaction. These include devices for capturing pointing and gestures, dedicated handheld devices, support for touch, and the use of collaborative actions involving input from multiple people.

While the individual domains of visual analytics, immersion, and computer mediated collaboration each have a long and rich research history, their combination that gives rise to CIA is very new and so there are significant opportunities for future work. As discussed, fewer than three percent of the papers published in the main visualization conferences even address collaboration. The chapter conclusion identifies a number of topics for research including using VR for CIA, developing methods for distant CIA, exploring the CIA design space, asynchronous collaboration, and methods for evaluating CIA systems. Perhaps most importantly is research in the area of multi-user interaction. Interaction modalities that may work well in immersive environments in general, are not necessarily appropriate for complex collaborative analysis work, that may last over several sessions, with participants connecting using different technologies. If and how technological asymmetry between colleagues can affect the quality of analysis work is still an open question.

Overall, Collaborative Immersive Analytics is an exciting new field with significant potential for improving how teams problem-solve, and presents many opportunities for ongoing research. CIA is at the same point that the field of visualization was at twenty years ago, and in the same way that visualization has become a key tool in the years since, we expect that CIA will dramatically change, and challenge, problem solving in the future.

\section{References}

1. Anslow, C.: Reflections on collaborative software visualization in co-located environments. In: IEEE International Conference on Software Maintenance and Evolution (ICSME). pp. 645-650. IEEE (2014)

2. Anwar, A., Klein, B., Berger, M., Arisona, S.M.: Value Lab Asia: A space for physical and virtual interdisciplinary research and collaboration. In: 19th International Conference on Information Visualisation (IV). pp. 348-353. IEEE (2015)

3. Arglasses(2016), http://arglassesguide.com/ 
4. Argelaguet Sanz, F., Andujar, C.: A survey of 3D object selection techniques for virtual environments. Computers and Graphics 37(3), 121-136(2013), https: //hal.archives-ouvertes.fr/hal-00907787

5. Badam, S.K., Elmqvist, N.: Polychrome: A cross-device framework for collaborative web visualization. In: Proceedings of the Ninth ACM International Conference on Interactive Tabletops and Surfaces. pp. 109-118. ACM (2014)

6. Bai, H., Lee, G., Billinghurst, M.: Free-hand gesture interfaces for an augmented exhibition podium. In: Proceedings of the Annual Meeting of the Australian Special Interest Group for Computer Human Interaction. pp. 182-186. OzCHI '15, ACM, New York, NY, USA (2015)

7. Ballendat, T., Marquardt, N., Greenberg, S.: Proxemic interaction: Designing for a proximity and orientation-aware environment. In: ACM International Conference on Interactive Tabletops and Surfaces. pp. 121-130. ACM, New York, NY, USA (2010)

8. Belcher, D., Billinghurst, M., Hayes, S., Stiles, R.: Using augmented reality for visualizing complex graphs in three dimensions. In: Proceedings of the Second IEEE and ACM International Symposium on Mixed and Augmented Reality. pp. 84-93. IEEE (2003)

9. Benbunan-Fich, R., Hiltz, S.R., Turoff, M.: A comparative content analysis of faceto-face vs. asynchronous group decision making. Decision Support Systems 34(4), 457-469 (2003)

10. Benford, S., Fahlén, L.: A spatial model of interaction in large virtual environments. In: Proceedings of the Third Conference on European Conference on ComputerSupported Cooperative Work. pp. 109-124. ECSCW'93, Kluwer Academic Publishers, Norwell, MA, USA (1993)

11. Benko, H., Holz, C., Sinclair, M., Ofek, E.: NormalTouch and TextureTouch: Highfidelity $3 \mathrm{D}$ haptic shape rendering on handheld virtual reality controllers. In: Proceedings of the 29th Annual Symposium on User Interface Software and Technology. pp. 717-728. UIST '16, ACM, New York, NY, USA (2016)

12. Benko, H., Ishak, E.W., Feiner, S.: Collaborative mixed reality visualization of an archaeological excavation. In: Proceedings of the 3rd IEEE/ACM International Symposium on Mixed and Augmented Reality. pp. 132-140. IEEE Computer Society (2004)

13. Bezerianos, A., Isenberg, P.: Perception of visual variables on tiled wall-sized displays for information visualization applications. IEEE Transactions on Visualization and Computer Graphics 18(12), 2516-2525 (2012)

14. Bezerianos, A., Dragicevic, P., Balakrishnan, R.: Mnemonic rendering: An imagebased approach for exposing hidden changes in dynamic displays. In: Proceedings of the 19th Annual ACM Symposium on User Interface Software and Technology. pp. 159-168. UIST '06, ACM, New York, NY, USA (2006)

15. Bowman, D.A., Wingrave, C.A.: Design and evaluation of menu systems for immersive virtual environments. In: Proceedings of the Virtual Reality 2001 Conference (VR'01). pp. 149-. VR '01, IEEE Computer Society (2001)

16. Bradel, L., Endert, A., Koch, K., Andrews, C., North, C.: Large high resolution displays for co-located collaborative sensemaking: Display usage and territoriality. International Journal of Human-Computer Studies 71(11), 1078-1088 (2013)

17. Card, S.K., Mackinlay, J.D., Shneiderman, B.: Readings in Information Visualization: Using Vision to Think. Morgan Kaufmann (1999)

18. Carpendale, S., Diakopoulos, N., Henry Riche, N., Hurter, C.: Data-Driven Storytelling. Dagstuhl Reports 6(2), 1-27(2016), https://hal-enac.archives-ouvertes.fr/hal01348422 
19. Carter, S., Mankoff, J., Goddi, P.: Building connections among loosely coupled groups: Hebb's rule at work. Computer Supported Cooperative Work (CSCW) 13(3-4), 305-327 (2004)

20. Chandler, T., Cordeil, M., Czauderna, T., Dwyer, T., Glowacki, J., Goncu, C., Klapperstueck, M., Klein, K., Marriott, K., Schreiber, F., et al.: Immersive analytics. In: Big Data Visual Analytics (BDVA), 2015. pp. 1-8. IEEE (2015)

21. Chen, W., Ladeveze, N., Clavel, C., Mestre, D., Bourdot, P.: User cohabitation in multi-stereoscopic immersive virtual environment for individual navigation tasks. In: 2015 IEEE Virtual Reality (VR). pp. 47-54 (2015)

22. Chen, Y., Alsakran, J., Barlowe, S., Yang, J., Zhao, Y.: Supporting effective common ground construction in asynchronous collaborative visual analytics. In: IEEE Conference on Visual Analytics Science and Technology (VAST). pp. 101-110. IEEE (2011)

23. Chung, H., North, C., Self, J.Z., Chu, S., Quek, F.: VisPorter: facilitating information sharing for collaborative sensemaking on multiple displays. Personal and Ubiquitous Computing 18(5), 1169-1186 (2014)

24. Churchill, E.F., Snowdon, D.N., Munro, A.J.: Collaborative virtual environments: digital places and spaces for interaction. Springer Science \& Business Media (2012)

25. Clark, H.H., Brennan, S.E., et al.: Grounding in communication. Perspectives on Socially Shared Cognition 13(1991), 127-149 (1991)

26. Colaço, A., Kirmani, A., Yang, H.S., Gong, N.W., Schmandt, C., Goyal, V.K.: Mime: Compact, low power 3D gesture sensing for interaction with head mounted displays. In: Proceedings of the 26th Annual ACM Symposium on User Interface Software and Technology. pp. 227-236. UIST '13, ACM, New York, NY, USA (2013)

27. Cook, K.A., Thomas, J.J.: Illuminating the path: The research and development agenda for visual analytics. Tech. rep., Pacific Northwest National Laboratory (PNNL), Richland, WA (US) (2005)

28. Cordeil, M., Dwyer, T., Klein, K., Laha, B., Marriott, K., Thomas, B.H.: Immersive collaborative analysis of network connectivity: CAVE-style or head-mounted display? IEEE Transactions on Visualization and Computer Graphics 23(1), 441-450 (2017)

29. Craig, P., Huang, X., Chen, H., Wang, X., Zhang, S.: Pervasive information visualization: Toward an information visualization design methodology for multi-device co-located synchronous collaboration. In: IEEE International Conference on Computer and Information Technology; Ubiquitous Computing and Communications; Dependable, Autonomic and Secure Computing; Pervasive Intelligence and Computing (CIT/IUCC/DASC/PICOM). pp. 2232-2239. IEEE (2015)

30. Cruz-Neira, C., Sandin, D.J., DeFanti, T.A.: Surround-screen projection-based virtual reality: the design and implementation of the CAVE. In: Proceedings of the 20th Annual Conference on Computer Graphics and Interactive Techniques. pp. 135-142. ACM (1993)

31. Donalek, C., Djorgovski, S.G., Cioc, A., Wang, A., Zhang, J., Lawler, E., Yeh, S., Mahabal, A., Graham, M., Drake, A., et al.: Immersive and collaborative data visualization using virtual reality platforms. In: IEEE International Conference on Big Data (Big Data). pp. 609-614. IEEE (2014)

32. Dourish, P., Bellotti, V.: Awareness and coordination in shared workspaces. In: Proceedings of the 1992 ACM Conference on Computer-supported Cooperative Work. pp. 107-114. CSCW '92, ACM, New York, NY, USA(1992), http: //doi.acm.org/10.1145/143457.143468

33. Edelson, D., Pea, R., Gomez, L., et al.: Constructivism in the collaboratory. Constructivist Learning Environments: Case Studies in Instructional Design 151 (1996) 
34. Elias, M., Aufaure, M.A., Bezerianos, A.: Storytelling in visual analytics tools for business intelligence. In: IFIP Conference on Human-Computer Interaction. pp. 280-297. Springer (2013)

35. Ellis, S.E., Groth, D.P.: A collaborative annotation system for data visualization. In: Proceedings of the Working Conference on Advanced Visual Interfaces. pp. 411-414. ACM (2004)

36. Feiner, S., Macintyre, B., Höllerer, T.: A touring machine: Prototyping 3D mobile augmented reality systems for exploring the urban environment. In: Personal Technologies 1(4). pp. 74-81 (1997)

37. Flystick: http://www.ar-tracking.com/products/interaction/flystick2/ ([Online; accessed 05-Feb-2017])

38. Fraser, M., Benford, S., Hindmarsh, J., Heath, C.: Supporting awareness and interaction through collaborative virtual interfaces. In: Proceedings of the 12th Annual ACM Symposium on User Interface Software and Technology. pp. 27-36. UIST '99, ACM, New York, NY, USA (1999)

39. Gjerlufsen, T., Klokmose, C.N., Eagan, J., Pillias, C., Beaudouin-Lafon, M.: Shared substance: Developing flexible multi-surface applications. In: Proceedings of the SIGCHI Conference on Human Factors in Computing Systems. pp. 3383-3392. CHI '11, ACM, New York, NY, USA (2011)

40. Greenberg, S., Rounding, M.: The notification collage: posting information to public and personal displays. In: Proceedings of the SIGCHI Conference on Human Factors in Computing Systems. pp. 514-521. ACM (2001)

41. Gunawardena, C.N., Zittle, F.J.: Social presence as a predictor of satisfaction within a computer-mediated conferencing environment. American Journal of Distance Education 11(3), 8-26 (1997)

42. Gupta, K., Lee, G.A., Billinghurst, M.: Do you see what I see? the effect of gaze tracking on task space remote collaboration. IEEE Transactions on Visualization and Computer Graphics 22(11), 2413-2422 (2016)

43. Gutwin, C., Greenberg, S.: Design for individuals, design for groups: tradeoffs between power and workspace awareness. In: Proceedings of the 1998 ACM Conference on Computer Supported Cooperative Work. pp. 207-216. ACM (1998)

44. Gutwin, C., Greenberg, S.: A descriptive framework of workspace awareness for real-time groupware. Computer Supported Cooperative Work 11(3), 411-446 (2002)

45. Hackathorn, R., Margolis, T.: Immersive analytics: Building virtual data worlds for collaborative decision support. In: 2016 Workshop on Immersive Analytics (IA). pp. 44-47(March 2016) doi: 10.1109/IMMERSIVE. 2016.7932382

46. Hauber, J., Regenbrecht, H., Billinghurst, M., Cockburn, A.: Spatiality in videoconferencing: trade-offs between efficiency and social presence. In: Proceedings of the 2006 20th Anniversary Conference on Computer Supported Cooperative Work. pp. 413-422. ACM (2006)

47. Heath, C., Luff, P.: Disembodied conduct: communication through video in a multimedia office environment. In: Proceedings of the SIGCHI Conference on Human Factors in Computing Systems. pp. 99-103. ACM (1991)

48. Heer, J., Agrawala, M.: Design considerations for collaborative visual analytics. Information Visualization 7(1), 49-62 (2008)

49. Heer, J., Viégas, F.B., Wattenberg, M.: Voyagers and voyeurs: supporting asynchronous collaborative information visualization. In: Proceedings of the SIGCHI Conference on Human Factors in Computing Systems. pp. 1029-1038. ACM (2007)

50. Hendrix, T.D., Cross II, J.H., Maghsoodloo, S., McKinney, M.L.: Do visualizations improve program comprehensibility? experiments with control structure diagrams for java. In: ACM SIGCSE Bulletin. vol. 32, pp. 382-386. ACM (2000) 
51. Hill, G.W.: Group versus individual performance: Are N+1 heads better than one? Psychological Bulletin 91(3), 517 (1982)

52. Hiltz, S.R.: The virtual classroom: Learning without limits via computer networks. Intellect Books (1994)

53. Hutterer, P., Close, B.S., Thomas, B.H.: Supporting mixed presence groupware in tabletop applications. In: First IEEE International Workshop on Horizontal Interactive Human-Computer Systems (TableTop 2006). pp. 8-pp. IEEE (2006)

54. Imai, T., Johnson, A.E., Leigh, J., Pape, D.E., DeFanti, T.A.: The virtual mail system. In: Proceedings of IEEE Virtual Reality. p. 78. IEEE (1999)

55. Imai, T., Qiu, Z., Behara, S., Tachi, S., Aoyama, T., Johnson, A., Leigh, J.: Overcoming time-zone differences and time management problem with tele-immersion. In: The Proceedings of INET. pp. 18-21 (2000)

56. Isenberg, P., Bertini, E., Lam, H., Plaisant, C., Carpendale, S.: Empirical studies in information visualization: Seven scenarios. IEEE Transactions on Visualization \& Computer Graphics 18, 1520-1536 (2012)

57. Isenberg, P., Bezerianos, A., Henry, N., Carpendale, S., Fekete, J.D.: CoCoNutTrix: Collaborative retrofitting for information visualization. IEEE Computer Graphics and Applications 29(5), 44-57(2009), https://hal.inria.fr/hal-00690020

58. Isenberg, P., Elmqvist, N., Scholtz, J., Cernea, D., Ma, K.L., Hagen, H.: Collaborative visualization: definition, challenges, and research agenda. Information Visualization 10(4), 310-326 (2011)

59. Isenberg, P., Fisher, D.: Collaborative brushing and linking for co-located visual analytics of document collections. In: Proceedings of the 11th Eurographics / IEEE VGTC Conference on Visualization. pp. 1031-1038. EuroVis'09, The Eurographs Association \&\#38; John Wiley \&\#38; Sons, Ltd., Chichester, UK (2009)

60. Isenberg, P., Fisher, D., Ringel Morris, M., Inkpen, K., Czerwinski, M.: An exploratory study of co-located collaborative visual analytics around a tabletop display. In: Proceedings of Visual Analytics Science and Technology (VAST). pp. 179-186. IEEE, Salt Lake City, UT, United States(2010), https://hal.inria.fr/inria-00587236, received an honorable mention at VAST 2010

61. Ishii, H., Kobayashi, M., Arita, K.: Iterative design of seamless collaboration media. Communications of the ACM 37(8), 83-97 (1994)

62. Jakobsen, M.R., Hornbaek, K.: Up close and personal: Collaborative work on a high-resolution multitouch wall display. ACM Transactions on Computer-Human Interaction 21(2), 11:1-11:34 (2014)

63. Jakobsen, M.R., Sahlemariam Haile, Y., Knudsen, S., Hornbæk, K.: Information visualization and proxemics: Design opportunities and empirical findings. IEEE Transactions on Visualization and Computer Graphics 19(12), 2386-2395 (2013)

64. Jansen, Y., Dragicevic, P., Fekete, J.D.: Tangible remote controllers for wall-size displays. In: Proceedings of the SIGCHI Conference on Human Factors in Computing Systems. pp. 2865-2874. CHI '12, ACM, New York, NY, USA (2012)

65. Johansen, R.: Groupware: Computer support for business teams. The Free Press (1988)

66. Johnson, A., Leigh, J.: Tele-immersive collaboration in the CAVE research network. In: Collaborative Virtual Environments. pp. 225-243. Springer (2001)

67. Johnson, A., Roussos, M., Leigh, J., Vasilakis, C., Barnes, C., Moher, T.: The nice project: Learning together in a virtual world. In: Proceedings of the IEEE Virtual Reality Annual International Symposium. pp. 176-183. IEEE (1998)

68. Johnson, C.: Top scientific visualization research problems. IEEE Computer Graphics and Applications 24(4), 13-17 (2004) 
69. Keates, S., Robinson, P.: The use of gestures in multimodal input. In: Proceedings of the Third International ACM Conference on Assistive Technologies. pp. 35-42. Assets '98, ACM, New York, NY, USA (1998)

70. Kim, K., Javed, W., Williams, C., Elmqvist, N., Irani, P.: Hugin: A framework for awareness and coordination in mixed-presence collaborative information visualization. In: ACM International Conference on Interactive Tabletops and Surfaces. pp. 231240. ACM (2010)

71. Kinnect(2016), https://developer.microsoft.com/en-us/windows/kinect

72. Klapperstueck, M., Czauderna, T., Goncu, C., Glowacki, J., Dwyer, T., Schreiber, F., Marriott, K.: ContextuWall: Multi-site collaboration using display walls. Journal of Visual Languages and Computing 46, 35 - 42(2018) doi: 10.1016/j.jvlc.2017.10. 002

73. Kratz, S., Ferriera, F.R.: Immersed remotely: Evaluating the use of head mounted devices for remote collaboration in robotic telepresence. In: 2016 25th IEEE International Symposium on Robot and Human Interactive Communication (RO-MAN). IEEE (aug 2016)

74. Leapmotion(2016), https://www.leapmotion.com/

75. Lee, B., Henry Riche, N., Isenberg, P., Carpendale, S.: More than telling a story: A closer look at the process of transforming data into visually shared stories. IEEE Computer Graphics and Applications 35(5), 84-90 (2015)

76. Leigh, J., Brown, M.D.: Cyber-commons: merging real and virtual worlds. Communications of the ACM 51(1), 82-85 (2008)

77. Leigh, J., Johnson, A.E., Brown, M., Sandin, D.J., DeFanti, T.A.: Visualization in teleimmersive environments. Computer 32(12), 66-73 (1999)

78. Liu, C., Chapuis, O., Beaudouin-Lafon, M., Lecolinet, E.: Shared interaction on a wall-sized display in a data manipulation task. In: Proceedings of the 34th International Conference on Human Factors in Computing Systems. pp. 2075-2086. CHI '16, ACM (2016)

79. Liu, C., Chapuis, O., Beaudouin-Lafon, M., Lecolinet, E.: CoReach: Cooperative gestures for data manipulation on wall-sized displays. In: ACM (ed.) Proceedings of the 35th International Conference on Human Factors in Computing Systems. CHI '17, Denver, United States(2017), https://hal.archives-ouvertes.fr/hal-01437091

80. Mahyar, N., Sarvghad, A., Tory, M.: Note-taking in co-located collaborative visual analytics: Analysis of an observational study. Information Visualization 11(3), 190 $204(2012)$

81. Mahyar, N., Tory, M.: Supporting communication and coordination in collaborative sensemaking. IEEE Transactions on Visualization and Computer Graphics 20(12), 1633-1642 (2014)

82. Marai, G.E., Forbes, A.G., Johnson, A.: Interdisciplinary immersive analytics at the electronic visualization laboratory: Lessons learned and upcoming challenges (2018)

83. Mark, G., Carpenter, K., Kobsa, A.: Are there benefits in seeing double? A study of collaborative information visualization. In: CHI'03 Extended Abstracts on Human Factors in Computing Systems. pp. 840-841. ACM (2003)

84. McCarthy, J.F., Costa, T.J., Liongosari, E.S.: Unicast, outcast \& groupcast: Three steps toward ubiquitous, peripheral displays. In: International Conference on Ubiquitous Computing. pp. 332-345. Springer (2001)

85. McGrath, W., Bowman, B., McCallum, D., Hincapié-Ramos, J.D., Elmqvist, N., Irani, P.: Branch-explore-merge: Facilitating real-time revision control in collaborative visual exploration. In: Proceedings of the 2012 ACM International Conference on 
Interactive Tabletops and Surfaces. pp. 235-244. ITS '12, ACM, New York, NY, USA (2012)

86. Milgram, P., Kishino, F.: A taxonomy of mixed reality visual displays. IEICE Transactions on Information and Systems 77(12), 1321-1329 (1994)

87. Millán, J.d.R.: Brain-computer interfaces. Tech. rep., The MIT Press (2002)

88. Moraes, A.C., Eler, D.M., Brega, J.R.: Collaborative information visualization using a multi-projection system and mobile devices. In: 18th International Conference on Information Visualisation (IV). pp. 71-77. IEEE (2014)

89. Morozov, M., Gerasimov, A., Fominykh, M.: vAcademia-educational virtual world with 3D recording. In: 2012 International Conference on Cyberworlds (CW). pp. 199-206. IEEE (2012)

90. Morris, M.R., Huang, A., Paepcke, A., Winograd, T.: Cooperative gestures: Multiuser gestural interactions for co-located groupware. In: Proceedings of the SIGCHI Conference on Human Factors in Computing Systems. pp. 1201-1210. CHI '06, ACM, New York, NY, USA (2006)

91. Morris, M.R., Lombardo, J., Wigdor, D.: Wesearch: Supporting collaborative search and sensemaking on a tabletop display. In: Proceedings of the 2010 ACM Conference on Computer Supported Cooperative Work. pp. 401-410. CSCW '10, ACM, New York, NY, USA (2010)

92. Nacenta, M.A., Sallam, S., Champoux, B., Subramanian, S., Gutwin, C.: Perspective Cursor: Perspective-based interaction for multi-display environments. In: Proceedings of the SIGCHI Conference on Human Factors in Computing Systems. pp. 289-298. CHI '06, ACM, New York, NY, USA (2006)

93. Nancel, M., Pietriga, E., Chapuis, O., Beaudouin-Lafon, M.: Mid-air pointing on ultra-walls. ACM Transactions on Computer-Human Interaction 22(5), 62 pages (2015)

94. Nassani, A., Bai, H., Lee, G., Billinghurst, M.: Tag It!: AR annotation using wearable sensors. In: SIGGRAPH Asia 2015 Mobile Graphics and Interactive Applications. pp. 12:1-12:4. SA '15, ACM, New York, NY, USA (2015)

95. Nature specials "big data" (2008)

96. Nguyen, T.T.H., Duval, T., Fleury, C.: Guiding Techniques for Collaborative Exploration in Multi-Scale Shared Virtual Environments. In: GRAPP International Conference on Computer Graphics Theory and Applications. pp. 327-336. Barcelona Spain(2013), https://hal.archives-ouvertes.fr/hal-00755313

97. Olwal, A., Frykholm, O., Groth, K., Moll, J.: Design and evaluation of interaction technology for medical team meetings. In: Human-Computer Interaction INTERACT 2011. pp. 505-522. Springer Berlin Heidelberg (2011)

98. Pauchet, A., Coldefy, F., Lefebvre, L., Picard, S., Bouguet, A., Perron, L., Guerin, J., Corvaisier, D., Collobert, M.: Mutual awareness in collocated and distant collaborative tasks using shared interfaces. Human-Computer Interaction-Interact 2007 pp. 59-73 (2007)

99. Poole, A., Ball, L.J.: Eye tracking in hci and usability research. Encyclopedia of Human Computer Interaction 1, 211-219 (2006)

100. Pousman, Z., Stasko, J.: A taxonomy of ambient information systems: four patterns of design. In: Proceedings of the Working Conference on Advanced Visual Interfaces. pp. 67-74. ACM (2006)

101. Prince, S., Cheok, A.D., Farbiz, F., Williamson, T., Johnson, N., Billinghurst, M., Kato, H.: 3DD Live: Real time interaction for mixed reality. In: Proceedings of the 2002 ACM Conference on Computer Supported Cooperative Work. pp. 364-371. CSCW '02, ACM, New York, NY, USA (2002) 
102. Prouzeau, A., Bezerianos, A., Chapuis, O.: Evaluating multi-user selection for exploring graph topology on wall-displays. IEEE Transactions on Visualization and Computer Graphics p. 14 pages(2016), https://hal.archives-ouvertes.fr/hal-01348578

103. Regenbrecht, H., Haller, M., Hauber, J., Billinghurst, M.: Carpeno: interfacing remote collaborative virtual environments with table-top interaction. Virtual Reality 10(2), 95-107 (2006)

104. Rice, R.E.: Computer-mediated communication and organizational innovation. Journal of Communication 37(4), 65-94 (1987)

105. Robinson, A.C.: Collaborative synthesis of visual analytic results. In: 2008 IEEE Symposium on Visual Analytics Science and Technology. pp. 67-74 (2008)

106. Robinson, P., Tuddenham, P.: Distributed tabletops: Supporting remote and mixedpresence tabletop collaboration. In: Second Annual IEEE International Workshop on Horizontal Interactive Human-Computer Systems (TABLETOP'07). pp. 19-26. IEEE (2007)

107. Satyanarayan, A., Heer, J.: Authoring narrative visualizations with ellipsis. Computer Graphics Forum 33(3), 361-370 (2014)

108. Sawyer, S., Farber, J., Spillers, R.: Supporting the social processes of software development. Information Technology \& People 10(1), 46-62 (1997)

109. Schmalstieg, D., Fuhrmann, A., Hesina, G., Szalavári, Z., Encarnaçao, L.M., Gervautz, M., Purgathofer, W.: The studierstube augmented reality project. Presence: Teleoperators and Virtual Environments 11(1), 33-54 (2002)

110. Seifert, J., Simeone, A., Schmidt, D., Holleis, P., Reinartz, C., Wagner, M., Gellersen, H., Rukzio, E.: Mobisurf: Improving co-located collaboration through integrating mobile devices and interactive surfaces. In: Proceedings of the 2012 ACM International Conference on Interactive Tabletops and Surfaces. pp. 51-60. ITS '12, ACM, New York, NY, USA (2012)

111. Shaffer, E., Reed, D.A., Whitmore, S., Schaeffer, B.: Virtue: Performance visualization of parallel and distributed applications. Computer 32(12), 44-51 (1999)

112. Shneiderman, B.: Direct manipulation: A step beyond programming languages. Computer 16(8), 57-69 (1983)

113. Skog, T., Ljungblad, S., Holmquist, L.E.: Between aesthetics and utility: designing ambient information visualizations. In: IEEE Symposium on Information Visualization (INFOVIS 2003). pp. 233-240. IEEE (2003)

114. Snowdon, D., Grasso, A.: Diffusing information in organizational settings: learning from experience. In: Proceedings of the SIGCHI Conference on Human Factors in Computing Systems. pp. 331-338. ACM (2002)

115. Soares, A.G.M., dos Santos, C.G.R., Mendonça, S.D.P., Carneiro, N.J.S., Miranda, B.P., de Araújo, T.D.O., de Freitas, A.A., de Morais, J.M., Meiguins, B.S.: A review of ways and strategies on how to collaborate in information visualization applications. In: 20th International Conference on Information Visualisation (IV). pp. 81-87. IEEE (2016)

116. Sole, D., Wilson, D.: Storytelling in organizations: The power and traps of using stories to share knowledge in organizations. LILA (2002)

117. g speak: http://www.oblong.com/g-speak ([Online; accessed 05-Feb-2017])

118. Stasko, J., Catrambone, R., Guzdial, M., McDonald, K.: An evaluation of spacefilling information visualizations for depicting hierarchical structures. International Journal of Human-Computer Studies 53(5), 663-694 (2000)

119. Sun, G.D., Wu, Y.C., Liang, R.H., Liu, S.X.: A survey of visual analytics techniques and applications: State-of-the-art research and future challenges. Journal of Computer Science and Technology 28(5), 852-867 (2013) 
120. Tang, A., Boyle, M., Greenberg, S.: Understanding and mitigating display and presence disparity in mixed presence groupware. Journal of Research and Practice in Information Technology 37(2), 193-210 (2005)

121. Tang, A., Neustaedter, C., Greenberg, S.: Videoarms: embodiments for mixed presence groupware. In: People and Computers XX - Engage, pp. 85-102. Springer (2007)

122. Tang, J.C., Minneman, S.: VideoWhiteboard: video shadows to support remote collaboration. In: Proceedings of the SIGCHI Conference on Human Factors in Computing Systems. pp. 315-322. ACM (1991)

123. Teasley, S., Covi, L., Krishnan, M.S., Olson, J.S.: How does radical collocation help a team succeed? In: Proceedings of the 2000 ACM Conference on Computer Supported Cooperative Work. pp. 339-346. ACM (2000)

124. Terrenghi, L., Quigley, A., Dix, A.: A taxonomy for and analysis of multi-persondisplay ecosystems. Personal and Ubiquitous Computing 13(8), 583 (2009)

125. Thomas, J.J.: Illuminating the path: the research and development agenda for visual analytics (2005)

126. Thomas, J.J., Cook, K.A.: A visual analytics agenda. IEEE Computer Graphics and Applications 26(1), 10-13 (2006)

127. Tsandilas, T., Bezerianos, A., Jacob, T.: SketchSliders: Sketching widgets for visual exploration on wall displays. In: Proceedings of the 33rd Annual ACM Conference on Human Factors in Computing Systems. pp. 3255-3264. CHI '15, ACM, New York, NY, USA (2015)

128. Tse, E., Greenberg, S., Shen, C., Forlines, C., Kodama, R.: Exploring true multiuser multimodal interaction over a digital table. In: Proceedings of the 7th ACM Conference on Designing Interactive Systems. pp. 109-118. DIS '08, ACM, New York, NY, USA (2008)

129. Turoff, M., Hiltz, S.R., Bahgat, A.N., Rana, A.R.: Distributed group support systems. MIS quarterly pp. 399-417 (1993)

130. Veerasamy, A., Belkin, N.J.: Evaluation of a tool for visualization of information retrieval results. In: Proceedings of the 19th Annual International ACM SIGIR conference on Research and Development in Information Retrieval. pp. 85-92. ACM (1996)

131. Viegas, F.B., Wattenberg, M.: Communication-minded visualization: A call to action. IBM Systems Journal 45(4), 801 (2006)

132. Viegas, F.B., Wattenberg, M., Van Ham, F., Kriss, J., McKeon, M.: Manyeyes: a site for visualization at internet scale. IEEE Transactions on Visualization and Computer Graphics 13(6) (2007)

133. Vogel, D., Balakrishnan, R.: Interactive public ambient displays: transitioning from implicit to explicit, public to personal, interaction with multiple users. In Proceedings of the 17th Annual ACM Symposium on User Interface Software and Technology. pp. 137-146. ACM (2004)

134. Voida, S., Tobiasz, M., Stromer, J., Isenberg, P., Carpendale, S.: Getting practical with interactive tabletop displays: Designing for dense data, "fat fingers," diverse interactions, and face-to-face collaboration. In: Proceedings of the ACM International Conference on Interactive Tabletops and Surfaces. pp. 109-116. ITS '09, ACM, New York, NY, USA (2009)

135. Ware, C., Franck, G.: Evaluating stereo and motion cues for visualizing information nets in three dimensions. ACM Transactions on Graphics (TOG) 15(2), 121-140 (1996) 
136. Willett, W., Heer, J., Hellerstein, J., Agrawala, M.: CommentSpace: structured support for collaborative visual analysis. In: Proceedings of the SIGCHI conference on Human Factors in Computing Systems. pp. 3131-3140. ACM (2011)

137. Woolley, A.W., Chabris, C.F., Pentland, A., Hashmi, N., Malone, T.W.: Evidence for a collective intelligence factor in the performance of human groups. Science 330(6004), 686-688 (2010)

138. Yasojima, E.K.K., Meiguins, B.S., Meiguins, A.S.G.: Collaborative augmented reality application for information visualization support. In: 16th International Conference on Information Visualisation (IV). pp. 164-169. IEEE (2012)

139. Yoo, B., Han, J.J., Choi, C., Yi, K., Suh, S., Park, D., Kim, C.: 3D user interface combining gaze and hand gestures for large-scale display. In: CHI '10 Extended Abstracts on Human Factors in Computing Systems. pp. 3709-3714. CHI EA '10, ACM, New York, NY, USA (2010)

140. von Zadow, U., Büschel, W., Langner, R., Dachselt, R.: SleeD: Using a sleeve display to interact with touch-sensitive display walls. In: Proceedings of the Ninth ACM International Conference on Interactive Tabletops and Surfaces. pp. 129-138. ITS '14, ACM, New York, NY, USA (2014)

141. Zhao, J., Glueck, M., Isenberg, P., Chevalier, F., Khan, A.: Supporting Handoff in Asynchronous Collaborative Sensemaking Using Knowledge-Transfer Graphs. IEEE Transactions on Visualization and Computer Graphics 24(1), 340-350(2018), https://hal.inria.fr/hal-01565560

142. Zhu, H.: From WYSIWIS to WISINWIS: role-based collaboration. In: 2004 IEEE International Conference on Systems, Man and Cybernetics. vol. 6, pp. 5441-5446. IEEE (2004)

143. Zimmer, B., Kerren, A.: Ongrax: A web-based system for the collaborative visual analysis of graphs. Journal of Graph Algorithms and Applications 21(1), 5-27 (2017) 